\title{
Control of MEMS Vibration Modes With Pulsed Digital Oscillators: Part I-Theory
}

\author{
Elena Blokhina, Member, IEEE, Joan Pons, Jordi Ricart, Orla Feely, Fellow, IEEE, and \\ Manuel Dominguez Pumar, Member, IEEE
}

\begin{abstract}
The aim of this paper is to show that it is possible to excite selectively different mechanical resonant modes of a MEMS structure using pulsed digital oscillators (PDOs). This can be done by simply changing the working parameters of the oscillator, namely its sampling frequency or its feedback filter. A set of iterative maps is formulated to describe the evolution of the spatial modes between two sampling events in PDOs. With this lumped model, it is established that under some circumstances PDO bitstreams related to only one of the resonances can be obtained, and that in the anti-oscillation regions of the PDO the mechanical energy is absorbed into the electrical domain on average. The possibility of selecting for a given resonant frequency the oscillation and anti-oscillation behavior allows one to obtain oscillations at any given resonant mode of the MEMS structure.
\end{abstract}

Index Terms-Energy efficiency, microelectromechanical systems (MEMS), microresonators, multimode control, oscillators, sigma-delta modulation.

\section{LIST OF SYMBOLS}

$\begin{array}{ll}\langle a\rangle & a \text { Modulus } 1, a \in \mathbb{R} . \\ \lfloor a\rfloor & \text { Largest integer less or equal to } a, a \in \mathbb{R} . \\ z^{*} & \text { Complex conjugate of } z \in \mathbb{C} . \\ \operatorname{Re}(z) & \text { Real part of } z \in \mathbb{C} . \\ \operatorname{Im}(z) & \text { Imaginary part of } z \in \mathbb{C} . \\ \text { g.c.d. }(M, N) & \text { Greater common divider, } M, N \in \mathbb{N} .\end{array}$

\section{INTRODUCTION}

$\mathbf{T}$ HERE is a large set of applications based on MEMS working in resonance, such as the sensing of acceleration, pressure, mass change, etc. These resonant sensors often detect shifts of the resonant frequency or amplitude changes of MEMS structures put in resonance in response to an external stimulus [1]-[4]. In most cases, resonant MEMS exploit structures such as beams [3]-[6] or plates [2], [7] with additional supporting elements (springs, arms, etc.) that are excited in one of their mechanical modes.

Manuscript received June 22, 2009; revised September 25, 2009; accepted November 22, 2009. Date of publication February 05, 2010; date of current version August 11, 2010. This work was supported by the Spanish Government through the TEC2007-67951/MIC (FEDER) project and by Science Foundation Ireland. This paper was recommended by Associate Editor Z. Galias.

E. Blokhina and O. Feely are with the School of Electrical, Electronic and Mechanical Engineering, University College Dublin, Belfield, Dublin 4, Ireland.

J. Pons, J. Ricart, and M. Dominguez are with the Micro and Nano Technologies Group, Universitat Politécnica de Catalunya, Barcelona 08034, Spain (email: mpumar@eel.upc.es).

Digital Object Identifier 10.1109/TCSI.2009.2038541

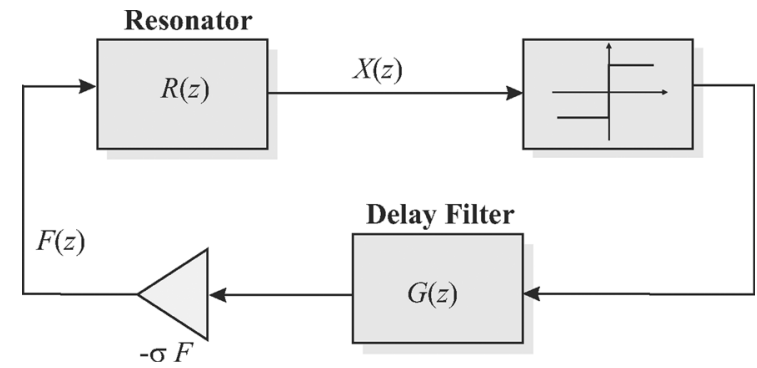

Fig. 1. General single feedback topology of the pulsed digital oscillator. In this paper $\mathrm{G}(\mathrm{z})$ is a delay filter $\left(G(z)=z^{-L-1}\right)$.

With the development of the MEMS technology, a lot of efforts have been made to improve the sensitivity of resonant sensors (see [8] and papers cited there). In most cases, the sensitivity of such sensors depends on the resonant frequency of the MEMS structure, which is usually excited in one of its mechanical resonant modes. Most of the methods that have been proposed to improve the sensitivity of gravimetric (or mass change) resonant sensors are based on increasing their operating frequency. For instance, parametric resonance amplification is an example of an efficient technique [9]-[12] that allows the excitation of the same mechanical structure at a higher frequency to improve sensitivity. In gravimetric sensors, the use of higher order modes generally serves to increase the sensitivity to mass changes, whereas it is desired in gyroscopes to avoid the activation of certain resonant modes of the inertial mass [13]. Moreover, the activation of higher vibration modes to increase performance has been also reported in atomic force microscopes [14], [15] and in piezoelectric sensors and actuators [16], where electrodes have been specifically designed to activate certain modes.

Thus, depending on the specific application, the selective activation of different spatial vibration modes of a mechanical resonator can be one way to improve performance for a large number of MEMS sensors [17]. The purpose of this paper is to show that pulsed digital oscillators (PDOs) [18], [19], may be a tool to selectively excite self-sustained resonant modes in MEMS structures.

PDOs are simple circuits that allow linear resonators to maintain self-sustained oscillations. The PDO general single-feedback topology is depicted in Fig. 1. The circuit topology consists of a resonator, a 1-bit quantizer (sign function), and a simple digital feedback filter, $G(z)$. The position of the MEMS resonator is evaluated at each sampling time, and short pulses of force are applied to the resonator. These circuits allow changes in the resonant frequency of the resonator to be monitored simply by processing the binary sequence generated at their output [20], [21]. The use of a pulsed actuation with constant amplitude and simplified position/velocity sensing 
requirements overcomes some of the nonlinearities usually found in MEMS with standard actuation techniques. This is clearly the case for the example of thermoelectric actuation, where the applied force is proportional to the square of the applied voltage or current. The same approach can also be used to the electrostatic actuation case, if the variation of the electrode gap is negligible (small signal displacement).

In order to analyze the dynamics of PDOs working with MEMS resonators with more than one resonance it is convenient to obtain the iterative map of the dynamical system, using a lumped model for the MEMS structure. The dynamics of MEMS structures that display predominately one-dimensional behavior, such as cantilevers or beams, are described through 1D partial differential equations (PDEs) [22]. A common strategy is to reduce distributed systems in the form of PDEs to lumped systems in the form of ordinary differential equations (ODEs) [23]. This way, instead of using a PDE, this device can be described by a set of mass-spring-damper coupled differential equations, the typical and yet effective way to model the dynamics of a variety of MEMS [7], [19], [20], [24]-[27]. This is an important result that will allow us to link the MEMS geometry with the iterative map of the PDO working with the resonator. We start with the partial differential equation (Section II) that describes the transversal deflections of a beam [22], [28]. Then a system of ordinary differential equations, coupled via the external driving, is obtained. Based on the pulsed nature of the actuation, in Section III a set of iterative maps is obtained to describe the evolution of the spatial modes between two sampling events.

Once the lumped model has been obtained, the iterative map of the PDO working with a resonator with several resonances is found in Section III. The objective of Section IV is to show that under some circumstances the PDO is able to excite only one of the resonances of the resonator. In this case the bitstream at the output of the oscillator is related exclusively to that resonance. It will be shown that this behavior may depend on the initial condition of the resonator. This implies that in order to select a given resonance another mechanism is needed. In [25] it was shown that under some circumstances the actuation of PDOs on linear resonators may, on average, extract energy out of the resonator. This mechanism is for the first time analyzed and characterized in a general way in Section V. It will be used as a tool to generate selective oscillation in MEMS resonators in part II of the paper.

\section{Analysis of the Vibration Modes of Elastic Beams}

In order to obtain a suitable lumped model to generate the iterative map of PDOs actuating resonators with more than one resonance, it is necessary to analyze the mechanical resonances of the structure. The general problem of transversal vibrations on elastic beams has been considered, for instance, in books [28], [22], including direct applications to MEMS in the latter book. A number of papers have studied beam vibrations in the context of MEMS [23], [29]-[33].

The aim of this section is to derive a reduced-order model of a specific MEMS cantilever with the given external driving using one of the techniques described in review [23] and successfully applied to MEMS in the above-mentioned papers. This is a required step in order to obtain the lumped model, which later

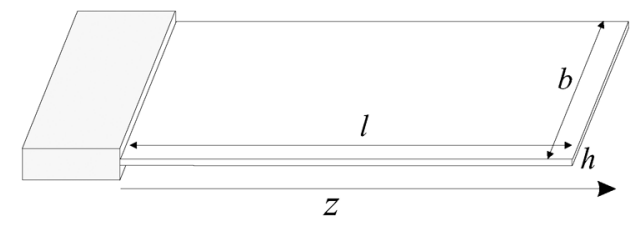

Fig. 2. Schematic drawing of the clamped-free beam in the PDO.

will allow the generation of the PDO iterative map actuating the MEMS resonator variables. We will start from a general PDE that describes transversal deflections of a cantilever and apply a strategy based on discretizing the initial distributed system by means of a set of spatial eigenmode functions $\psi_{i}(x, y, z)$, which in the general case is infinite. As a result, we will obtain a set of ordinary differential equations that represent the dynamics of each spatial mode as the mass-spring-damper equation with appropriate parameters.

Let us emphasize that in contrast to [30]-[32] that examine MEMS devices with electrostatic actuation, we will focus on a system that is subjected to a different type of excitation (driving by short pulses) and discuss the impact of such type of driving on mechanical modes generated in beams.

According to this, we assume that the MEMS cantilever is a clamped-free beam shown in Fig. 2 that is excited by the external force $F(\xi, \tau)$. The dimensionless equation and the boundary conditions that describe the transverse vibrations of the beam are as follows [28]:

$\frac{\partial^{4} u}{\partial \xi^{4}}+\frac{\partial^{2} u}{\partial \tau^{2}}+\gamma \frac{\partial u}{\partial \tau}=F(\xi, \tau)$

$u(0, \tau)=\frac{\partial u(0, \tau)}{\partial \xi}=0$ and $\frac{\partial^{2} u(1, \tau)}{\partial \xi^{2}}=\frac{\partial^{3} u(1, \tau)}{\partial \xi^{3}}=0$

where $u(\xi, \tau)$ is the transverse displacement at the position $\xi$ and time $\tau$. In (1), the dimensionless coordinate along the beam axis $\xi$, time $\tau$ and the dissipation parameter due to viscous damping $\gamma$ are defined through the dimensional ones $z, t$, and $c$

$$
\xi=z / l, \quad \tau=\frac{t}{l^{2}} \sqrt{\frac{E I}{\rho A}}, \quad \gamma=\frac{c l^{2}}{\sqrt{\rho A E I}}
$$

where $E$ is the Young's modulus, $I$ is the moment of inertia of the cross-section (for beams with a rectangular cross-section $\left.I=b h^{3} / 12\right), \rho$ is the density, $A$ is the area of the cross section, $c$ is the dissipation coefficient, $l$ is the length of the beam, $b$ is its width and $h$ is its thickness.

Next, the continuous equation (1) is discretized using the truncated set of linear mode shapes [23], [34]

$$
u(\xi, \tau)=\sum_{i=1}^{M} x_{i}(\tau) \psi_{i}(\xi)
$$

where $x_{i}(\tau)$ are time-dependent functions and $\psi_{i}(\xi)$ are the spatial mode shapes. For a clamped-free beam, these functions are [28]:

$\psi_{i}(\xi)=\cosh \left(\Omega_{i} \xi\right)-\cos \left(\Omega_{i} \xi\right)+\nu_{i}\left(\sin \left(\Omega_{i} \xi\right)-\sinh \left(\Omega_{i} \xi\right)\right)$

where $\nu_{i}=\left(\cos \Omega_{i}+\cosh \Omega_{i}\right) /\left(\sin \Omega_{i}+\sinh \Omega_{i}\right)$, and $\Omega_{i}$ are the roots of the equation

$$
\cos \Omega \cosh \Omega=-1 .
$$




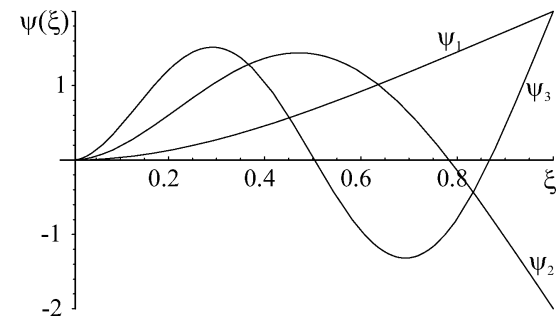

Fig. 3. Functions $\psi_{1,2,3}(\xi)$ versus the axial coordinate $\xi$.

The first three functions $\psi_{1,2,3}(\xi)$ (calculated according to (5) for the first three solutions of (6)) are shown in Fig. 3 as functions of the axial coordinate. Later in the paper, the vibrations that correspond to these functions will be called spatial modes with spatial frequencies $\Omega_{i}$.

Substituting (4) into (1) yields

$$
\sum_{i=1}^{M}\left(\ddot{x}_{i} \psi_{i}+\gamma \dot{x}_{i} \psi_{i}+x_{i} \psi^{\mathrm{IV}}\right)=F(\xi, \tau)+\varepsilon_{r}
$$

where the overdot notation denotes differentiation with respect to time, and the superscript 'IV' denotes the fourth derivative with respect to position. According the Galerkin procedure, we require that the residue term $\varepsilon_{r}$ be orthogonal to each eigenmode $\psi_{i}$. Multiplying the latter equation by $\psi_{j}$, integrating over the beam domain and taking into account that

$$
\int_{0}^{1} \psi_{i}^{2} d \xi=1, \int_{0}^{1} \psi_{i} \psi_{j} d \xi=\delta_{i j}, \int_{0}^{1} \psi_{i}^{I V} \psi_{j} d \xi=\Omega_{i}^{4} \delta_{i j}
$$

where $\delta_{i j}$ is the Kronecker delta, we obtain the system of ordinary differential equations

$$
\ddot{x}_{i}+\gamma \dot{x_{i}}+\Omega_{i}^{4} x_{i}=F_{i}(\tau), \quad i=1, \ldots, M .
$$

Note that the system (9) presents each spatial mode as the massspring-damper system with appropriate parameters such as the dissipation parameter $\gamma$ and the temporal frequency $\omega_{0 i}=\Omega_{i}^{2}$. Using that set of equations allows us to describe the evolution of spatial eigenmodes separately from each other and to obtain the overall solution of the problem in the form of a superposition of the modes. The functions $F_{i}$ in the right-hand side of the equations are the "projections" of the external force onto each eigenmode. They define what quantity of the force is transmitted to excite the oscillations of a mode:

$$
F_{i}(\tau)=\int_{0}^{1} \psi_{i}(\xi) F(\xi, \tau) d \xi .
$$

Using the expressions (3) that establish the correlations between dimension and dimensionless parameters, the radial and cyclic dimensional temporal frequencies in (9) are

$$
\omega_{0 i}=\frac{\Omega_{i}^{2}}{l^{2}} \sqrt{\frac{E I}{\rho A}}, \quad f_{0 i}=\frac{\Omega_{i}^{2}}{2 \pi l^{2}} \sqrt{\frac{E I}{\rho A}}
$$

and they are proportional to squared spatial frequencies $\Omega_{i}$.

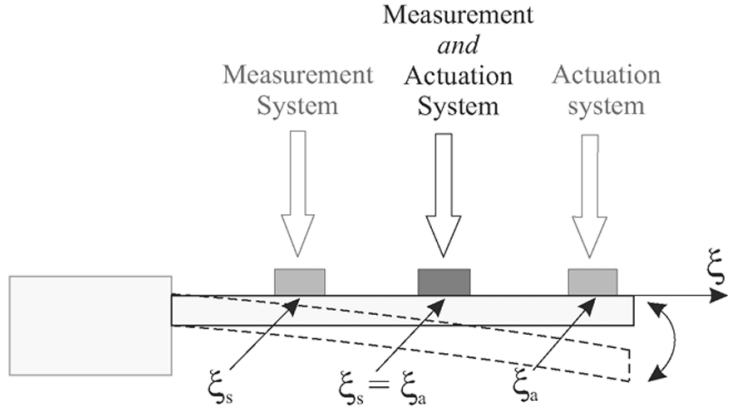

Fig. 4. Schematic drawing of the microbeam. The figure shows possible positions of the measurement $\left(\xi_{s}\right)$ and actuation system $\left(\xi_{a}\right)$.

The first four frequencies $\Omega_{i}$ calculated as the roots of (6) are as follows:

$$
\begin{aligned}
& \Omega_{1}=1.8751, \quad \Omega_{2}=4.69409 \\
& \Omega_{3}=7.85479, \quad \Omega_{4}=10.9955 .
\end{aligned}
$$

Now let us return to the PDO and rewrite the system of (9) for our specific case. To actuate the resonator, short pulses of force $\left\{+F_{0},-F_{0}\right\}$ are applied to it [18], with the sign of the pulses defined by the sign $\sigma$ of the gain in the feedback loop and on the position of the resonator. (For the resonator of the paper, we assume that we actuate the system by delta-pulses.) Position sensing is required only at the fixed time instants defined by the sampling frequency $f_{s}$. At these moments, the sensing system defines whether the resonator position is above or below the steady-state position. The actuation and sensing points in the cantilever can be placed in the same place or separated (see, e.g., Fig. 4). Let us denote $\xi_{s}$ as the coordinate along the beam axis at which the position of the resonator is measured by the sensing system and $\xi_{a}$ as the coordinate at which the pulse of external driving is applied to the resonator by the actuating system.

Although we present the excitation in our model as a sequence of Dirac delta pulses with a constant amplitude, and this model has its limitations, we suppose that this approach could be applied to a wider class of realistic MEMS devices that utilises pulsed driving to excite or stabilize oscillation of a mechanical structure. Let us give some discussion here.

In practical realizations, force pulses $F(t)$ always have finite width $T_{p}$. However, if $f_{0} \cdot T_{p} \ll 1$ and $T_{p} \ll 1 / \varsigma$, where $f_{0}$ is the natural frequency of oscillations and $\varsigma$ is the normalised dissipation, then the displacement caused by this short pulse $\Delta x \approx 0$ and the change of velocity $\Delta v \approx \int_{\tau_{n}}^{\tau_{n}+T_{p}} F(t) d t$. Therefore, mathematically this system can be reduced to one that is driven by Dirac delta pulses. Note that many MEMS are characterized by a relatively high frequency of oscillations and a high $Q$-factor, and these assumptions are typically valid.

Secondly, electrostatic short pulse actuation in the form $F_{e}(t)=F_{l}(t) /\left(1-\Delta x(t) / x_{0}\right)^{2}$, where $F_{l}$ is the linearized part of the force, $\Delta x(t)$ is the displacement and $x_{0}$ is the capacitor gap, can be modeled to some extent as constant amplitude pulses if oscillations of the resonator are small. In this case, we can neglect the nonlinear part of the force in that formula. In addition, in [35], for an electrostatically driven system short pulses were used as a compensation to avoid instability. Both cases were studied, the linearized force $F_{l}$ and the nonlinear 
$F_{e}$, and comparison of results were given there, showing that at high sampling frequencies the linearized approach is valid.

Thus, the external driving force for a beam embedded in the loop of a PDO can be written in the following form:

$$
\begin{gathered}
F(\xi, \tau)=-\sigma F_{0} \sum_{n} \operatorname{sgn}\left(u\left(\xi_{s},(n-L-1) T_{s}\right)\right) \\
\times Q\left(\xi-\xi_{a}\right) \delta\left(\tau-n T_{s}\right)
\end{gathered}
$$

where $u(\xi, \tau)$ is the position of the resonator, $L+1$ is the number of delays in the feedback (see Fig. 1), $\sigma$ is the sign of the feedback loop, $f_{s}=\left(f_{s}^{*} / 2 \pi l^{2}\right) \cdot \sqrt{E I / \rho A}$ is the dimensionless sampling frequency ( $f_{s}^{*}$ is its dimensional value), $T_{s}=1 / f_{s}$ is the dimensionless interval of time between two impulses, $\delta(x)$ is the Dirac delta function and $\operatorname{sgn}(x)$ is the signum function. In the most general case, an impulse is applied to a finite area at the beam, and, therefore, the function $Q(\xi)$ represents the "density distribution" of the actuating force. In the simplified statement of the problem, this function can be replaced by the Dirac delta function, i.e., $Q(\xi)=\delta(\xi)$. The latter means that the pulse is localized at the point $\xi$.

Let us denote $\tau_{n}=n T_{s}$ as the $n$th instant of time at which we apply the force pulse. Using the general definition (10) and the specific form of the force (14), the functions $F_{i}(\tau)$ may be expressed in the following way:

$$
\begin{aligned}
F_{i}(\tau)= & -\sigma F_{0} \sum_{n} \int_{0}^{1} \psi_{i}(\xi) \operatorname{sgn}\left(u\left(\xi_{s}, \tau_{n-L-1}\right)\right) \\
& \times Q\left(\xi-\xi_{a}\right) \delta\left(\tau-\tau_{n}\right) d \xi \\
= & -\sigma F_{0 i} \sum_{n} b_{n-L-1} \delta\left(\tau-\tau_{n}\right)
\end{aligned}
$$

where the amplitude of the external driving for each mode is denoted by

$$
F_{0 i}=F_{0} \int_{0}^{1} \psi_{i}(\xi) Q\left(\xi-\xi_{a}\right) d \xi
$$

and the sequence of signs is introduced by

$$
b_{n}=\operatorname{sgn} \sum_{i=1}^{M} x_{i}\left(\tau_{n}\right) \psi_{i}\left(\xi_{s}\right)
$$

Equation (15) emphasises that the $i$ th spatial mode receives a portion of the external driving which is proportional to the value $\psi_{i}\left(\xi_{a}\right)$. Note that in the simple case $Q\left(\xi-\xi_{a}\right)=\delta\left(\xi-\xi_{a}\right)$, the expression (15) is reduced to

$$
F_{0 i}=F_{0} \psi_{i}\left(\xi_{a}\right)
$$

Equation (16) uses the truncated expansion (4) to express the sensed positions of the beam.

Now, (9) and (14)-(16) can be used to specify the equations that will describe the dynamics of the first $M$ spatial modes $(i=1, \ldots, M)$ in the PDO with $L+1$ delay blocks in the feedback loop:

$$
\ddot{x}_{i}+\gamma \dot{x}_{i}+\Omega_{i}^{4} x_{i}=-\sigma F_{0 i} \sum_{n} b_{n-L-1} \delta\left(\tau-\tau_{n}\right)
$$

where the definitions of $F_{0 i}$ and $b_{n}$ are given above. For simplicity, the expressions (16) can be replaced by the following one (on condition that $\psi_{1}\left(\xi_{s}\right)>0$ ):

$$
b_{n}=\operatorname{sgn}\left\{x_{1}\left(\tau_{n}\right)+\beta_{2} x_{2}\left(\tau_{n}\right)+\cdots+\beta_{M} x_{M}\left(\tau_{n}\right)\right\}
$$

where

$$
\beta_{i}=\psi_{i}\left(\xi_{s}\right) / \psi_{1}\left(\xi_{s}\right)
$$

Formula (20) introduces a new family of parameters in order to avoid always operating in terms of the spatial functions $\psi_{i}$. The parameters $\beta_{i}$ now define the geometry of the sensing system and the contribution of the $i$ th spatial mode to the overall position of the resonator.

In the same manner, we can introduce the parameters $\mu_{i}$ that defines the geometry of the actuating system and the impact of the external driving on the $i$ th mode excitation:

$$
\mu_{i}=\psi_{i}\left(\xi_{a}\right) / \psi_{1}\left(\xi_{a}\right), \quad F_{0 i}=\mu_{i} F_{01} .
$$

In the case of two resonances, (19), (20), and (21) can be simply written as $b_{n}=\operatorname{sgn}\left\{x_{1}\left(\tau_{n}\right)+\beta x_{2}\left(\tau_{n}\right)\right\}$ with $\beta=$ $\psi_{2}\left(\xi_{s}\right) / \psi_{1}\left(\xi_{s}\right)$ and $F_{02}=\mu F_{01}$ with $\mu=\psi_{2}\left(\xi_{a}\right) / \psi_{1}\left(\xi_{a}\right)$.

Now the reduced-order model of the specific PDO system is defined by the set of equations (18)-(21). In the obtained model, only two parameters, $\beta$ and $\mu$, fully describe all possible positions of the actuating and sensing of the PDO on the MEMS resonator.

\section{ITERATIVE SYSTEM FOR THE PDO}

In the previous section we have converted the PDE to an ODE and obtained the system of (18) that describes the evolution of spatial modes in the PDO. In this section we discuss how the ordinary differential equations can be further reduced to discrete-time iterative equations (maps).

The system (9) is subjected to pulsed excitation given by the expressions (14). Between two sampling instants, the resonator moves freely, and at each sampling event its velocity is instantaneously changed by the applied delta-pulse. Thus, for the sequence of variables that we extract from the mass-springdamper equations (9) at the sampling instants, we will be able to formulate iterative equations.

In order to do so, for each spatial mode with the index " $i$ " we introduce the normalized damping factor $\rho_{i}=\gamma /\left(2 \Omega_{i}^{2}\right)$ and the variable

$$
v_{i}(\tau)=-\frac{1}{\sqrt{1-\rho_{i}^{2}}}\left(\rho_{i} x_{i}(\tau)+\frac{\dot{x}_{i}(\tau)}{\Omega_{i}^{2}}\right) .
$$

Now we can rewrite the ordinary differential equations of the second order (9) in the symmetrical form

$$
\begin{aligned}
\frac{d}{d \tau}\left(\begin{array}{c}
x_{i}(\tau) \\
v_{i}(\tau)
\end{array}\right) & =\Omega_{i}^{2}\left(\begin{array}{cc}
-\rho_{i} & -\sqrt{1-\rho_{i}^{2}} \\
\sqrt{1-\rho_{i}^{2}} & -\rho_{i}
\end{array}\right)\left(\begin{array}{c}
x_{i}(\tau) \\
v_{i}(\tau)
\end{array}\right) \\
v_{i}\left(\tau_{n}+\right)-v_{i}\left(\tau_{n}-\right) & =\frac{\sigma F_{0 i}}{\Omega_{i}^{2} \sqrt{1-\rho_{i}^{2}}} b_{n-L-1} .
\end{aligned}
$$

The solution of (22) is given by

$\left(\begin{array}{l}x_{i}(\tau) \\ v_{i}(\tau)\end{array}\right)=\exp \left(-\Omega_{i}^{2} \rho_{i} \tau\right) \mathbf{R}\left(\Omega_{i}^{2} \tau \sqrt{1-\rho_{i}^{2}}\right)\left(\begin{array}{c}x_{i}\left(\tau_{0}\right) \\ v_{i}\left(\tau_{0}\right)\end{array}\right)$ 
where $\mathbf{R}(\theta)=\left(\begin{array}{cc}\cos \theta & -\sin \theta \\ \sin \theta & \cos \theta\end{array}\right)$ is the rotation matrix by the angle $\theta$. If we define the sequence $\left\{x_{i, n}, v_{i, n}\right\}=$ $\left\{x_{i}\left(\tau_{n}\right), v_{i}\left(\tau_{n}\right)\right\}$ as the extracted values of $x_{i}(\tau)$ and $v_{i}(\tau)$ at the sampling instants $\tau_{n}$, the set of iterative equations will have the following form:

$$
\left(\begin{array}{c}
x_{i, n+1} \\
v_{i, n+1}
\end{array}\right)=\alpha_{i} \mathbf{R}\left(2 \pi f_{i}\right)\left(\begin{array}{c}
x_{i, n} \\
v_{i, n}
\end{array}\right)+\left(\begin{array}{c}
0 \\
\zeta_{i}
\end{array}\right) b_{n-L}
$$

for $i=1, \ldots, M$. Note that the values $b_{n}$ are defined by (16). In the set (24), we introduce the following dimensionless parameters:

$$
\begin{aligned}
\rho_{i} & =\frac{\gamma}{2 \Omega_{i}^{2}}, \quad f_{i}=\frac{\Omega_{i}^{2}}{f_{s}} \sqrt{1-\rho_{i}^{2}} \\
\zeta_{i} & =\frac{\sigma F_{0 i}}{\Omega_{i}^{2} \sqrt{1-\rho_{i}^{2}}}, \quad \alpha_{i}=\exp \left(\frac{-2 \pi \rho_{i} f_{i}}{\sqrt{1-\rho_{i}^{2}}}\right)
\end{aligned}
$$

where $\rho_{i}$ is the dimensionless damping parameter, $f_{i}$ is the normalized sample ratio in terms of the paper [26] (or normalized frequency in terms of the paper [20], both terms will be used throughout the paper), $\zeta_{i}$ is the normalized increment (note that it depends on the sign of the feedback loop) and $\alpha_{i}$ is the contraction factor. In (24), the first letter of the index, i.e., $i$, refers to the number of a spatial mode, and the second letter, i.e., $n$, refers to the iteration number.

In a similar way to the previous section, we draw attention to the particular case of the first two spatial modes. It is very convenient to establish the correlation between the parameters that refer to the first and the second modes in explicit form. Therefore, we introduce the ratio of the spatial frequencies of the modes as follows:

$$
\nu=\Omega_{2} / \Omega_{1}
$$

and apply it to connect the set of parameters for the modes:

$$
\begin{aligned}
\rho_{2} & =\frac{\rho_{1}}{\nu^{2}} \\
f_{2} & =\nu^{2} f_{1} \frac{\sqrt{1-\rho_{1}^{2} / \nu^{4}}}{\sqrt{1-\rho_{1}^{2}}} \approx \nu^{2} f_{1} \\
\zeta_{2} & =\frac{\mu \zeta_{1}}{\nu^{2}} \frac{\sqrt{1-\rho_{1}^{2}}}{\sqrt{1-\rho_{1}^{2} / \nu^{4}}} \approx \frac{\mu \zeta_{1}}{\nu^{2}} \\
\alpha_{2} & =\alpha_{1}^{\sqrt{1-\rho_{1}^{2}} / \sqrt{1-\rho_{1}^{2} / \nu^{4}}} \approx \alpha_{1} .
\end{aligned}
$$

Note that from the onset, our theory has included such parameters as the frequencies of the eigenmodes. As a consequence, the parameters used for formulation of the iterative system cannot be considered as independent ones, i.e., the values of controlling parameters have to be changed according the expressions (27). For instance, in the experimental set the sampling frequency is measured with respect to the frequency of the fundamental mode (i.e., the first spatial mode with the lowest frequency), and so the sampling ratio $f_{1}$ is given as a "basic" parameter. The value of $f_{2}$ can be obtained simply by recalculating $f_{1}$ with the ratio $\nu$.

Equations (24) introduce the iterative system as a set of 2D iterative maps and operate with variables such as the position of the resonator $x_{n}$ and the variable $v_{n}$ proportional to the velocity of the resonator. Results presented in these variables are easier to understand since $x_{n}$ and $v_{n}$ have an obvious physical interpretation. However, it is convenient to work on the complex plane with appropriate normalization and introduce a set of complex 1-D maps instead of 2-D maps written in real variables. By defining the following complex variable

$$
u_{i, n}=x_{i, n}-j v_{i, n}
$$

the evolution of the system is described now by the complex map

$$
u_{i, n+1}=p_{i} u_{i, n}-j \zeta_{i} b_{n-L}
$$

where $p_{i}=\alpha_{i} \exp \left(-j 2 \pi f_{i}\right)$, and for definition of $\alpha_{i}, f_{i}$ and $\zeta_{i}$ see (25).

The system (24) contains two coupled subsets-iterative equations that describe the first spatial mode component by the variables $x_{1, n}$ and $v_{1, n}$ and the second spatial mode by the variables $x_{2, n}$ and $v_{2, n}$. Later in the paper, dynamics that are displayed by the first subset of (24) will be referred as the "first spatial mode" (or the first mode, eigenmode or resonance), and dynamics that are displayed by the second subset will be referred as the "second spatial mode" (or the second mode). Note that the real position of the system is defined by the superposition of these two components according to the formula (4).

\section{Control of Resonant Modes With PDOS}

The objective of this section is to study the behavior of PDOs working with resonators with more than one resonance. Now that we have established the possibility of extracting a lumped model of the PDE governing a given MEMS structure, it is possible to continue the line of work introduced in [20] to study the dynamics of PDOs. The first result will show that under some conditions, depending on the initial condition of the resonator, it is possible to obtain bitstreams at the output of the oscillator related only to one of the resonant frequencies of the resonator. It will be shown later that, at least in some cases, the oscillation frequency may generally depend on the initial condition of the resonator.

On the other hand, it has already been shown in [36] that, given a resonator with one resonance and a configuration of the oscillator, either the oscillator induces self-sustained oscillations on it, or, on the contrary, tends to cancel any oscillation by extracting energy until some limit cycle is reached near the origin. This behavior will be used to selectively activate one of the resonances of a resonator with more than one resonance. The objective of Section V will be the study of this "anti-oscillation" behavior.

\section{A. Iterative Map With Two Resonances}

Let us focus on the case with two resonances. We will assume that the feedback filter is of the form $G(z)=z^{-L-1}$. Using the general complex formula (28) to formulate the iterative equations we obtain the following equations:

$$
\begin{aligned}
u_{1, n+1} & =p u_{1, n}-j \zeta_{1} b_{n-L} \\
u_{2, n+1} & =q u_{2, n}-j \zeta_{2} b_{n-L} \\
b_{n} & =\operatorname{sgn}\left(\operatorname{Re}\left(u_{1, n}+\beta u_{2, n}\right)\right)
\end{aligned}
$$


where $\zeta_{i}$ are normalized forces (25), $p=\alpha_{1} \exp \left(-j 2 \pi f_{1}\right)$, $q=\alpha_{2} \exp \left(-j 2 \pi f_{2}\right)$ and $\beta$ depends on the MEMS geometry. In the case of a cantilever it would be defined as in (20).

The dynamical system associated with these equations may be defined in the following way:

$$
\begin{array}{ccc}
\mathbb{C}^{2} \times \mathcal{B}^{L} & \tau & \mathbb{C}^{2} \times \mathcal{B}^{L} \\
\left(u_{1}, u_{2}, b_{1}, \ldots, b_{L}\right) & \rightarrow & \left(p u_{1}-j \zeta_{1} b_{L}, q u_{2}-j \zeta_{1} b_{L},\right. \\
& & \left.\operatorname{sgn}\left(\operatorname{Re}\left(u_{1}+\beta u_{2}\right)\right), b_{1}, \ldots, b_{L-1}\right)
\end{array}
$$

where $\mathcal{B}=\{-1,1\}, u_{1}, u_{2} \in \mathbb{C}, b_{i} \in \mathcal{B}, 1 \leq i \leq L$. For $L=0$

$$
\tau: \begin{array}{ccc}
\mathbb{C}^{2} \\
\left(u_{1}, u_{2}\right)
\end{array} \quad \begin{gathered}
\left(p u_{1}-j \zeta_{1} \operatorname{sgn}\left(\operatorname{Re}\left(u_{1}+\beta u_{2}\right)\right), .\right. \\
\\
\end{gathered}
$$

Now, following a similar approach to [20], we define:

Definition 1: The projections $\pi_{0}: \mathbb{C}^{2} \times \mathcal{B}^{L} \rightarrow \mathbb{C}^{2}$ and $\pi_{i}: \mathbb{C} \times \mathcal{B}^{L} \rightarrow \mathcal{B}, 1 \leq i \leq L$ are defined as $\pi_{0}\left(\left(u_{1}, u_{2}, b_{1}, \ldots, b_{L}\right)\right)=\left(u_{1}, u_{2}\right)$, and $\pi_{i}\left(\left(u_{1}, u_{2}, b_{1}, \ldots, b_{L}\right)\right)=b_{i}$.

We will consider that $\tau^{0}(z), z \in \mathbb{C}^{2} \times \mathcal{B}^{L}$, is the identity and that $\tau^{n}(z)$ is the result of $n$ applications of the function $\tau$ to $z, n>0$. In order to simplify notation we further define:

Definition 2: The function $T_{n}(z): \mathbb{C}^{2} \times \mathcal{B}^{L} \rightarrow \mathbb{C}^{2}, n \geq 0$ is defined as $\pi_{0}\left(\tau^{n}(z)\right)$.

Definition 3: The function $s: \mathbb{C}^{2} \rightarrow \mathcal{B}$ is defined as $s\left(u_{1}, u_{2}\right)=\operatorname{sgn}\left(\operatorname{Re}\left(u_{1}+\beta u_{2}\right)\right)$.

Definition 4: In the case $L \geq 1$, the functions $b_{n}^{i}(z): \mathbb{C} \times$ $\mathcal{B}^{L} \rightarrow \mathcal{B}$ are defined as $\pi_{i}\left(\tau^{n}(z)\right), 1 \leq i \leq L, n \geq 0$. If $L=0$, $b_{n}^{0}(z)$ is defined as $s\left(T_{n}(z)\right), n \geq 0$.

If $L \geq 1$ the feedback loop of the oscillator ensures that $b_{n}^{i}(z)=s\left(T_{n-i}(z)\right), 1 \leq i \leq L, n-i \geq 0$. If $n-i<0, n \geq 0$, then $b_{n}^{i}(z)=\pi_{|n-i|}(z)$.

Now, for a given binary sequence (b) we may define the sequence $h_{n}(p, \mathbf{b})$ as:

$$
h_{n}(p, \mathbf{b})=-j p^{n-1} \sum_{i=0}^{n-1} p^{-i} b_{i-L}, \quad n>0
$$

with $h_{0}(p, \mathbf{b})=0, p \in \mathbb{C}$, and test for the admissibility of that binary sequence:

Lemma 1: Given a sequence (b) and $z_{0}=$ $\left(u_{1,0}, u_{2,0}, b_{-1}, \ldots, b_{-L}\right) \in \mathbb{C}^{2} \times \mathcal{B}^{L}$, if $s\left(p^{n} u_{1,0}+\right.$ $\left.\zeta_{1} h_{n}(p, \mathbf{b}), q^{n} u_{2,0}+\zeta_{2} h_{n}(q, \mathbf{b})\right)=b_{n}, n>0$, then $s\left(T_{n}(z)\right)=b_{n}, n>0$.

Proof: It is obvious since in this case:

$$
\begin{aligned}
T_{n}\left(z_{0}\right)= & \left(p^{n} u_{1,0}-j \zeta_{1} p^{n-1} \sum_{i=0}^{n-1} p^{-i} s\left(T_{n-L}\left(z_{0}\right)\right),\right. \\
& \left.q^{n} u_{2,0}-j \zeta_{2} q^{n-1} \sum_{i=0}^{n-1} q^{-i} s\left(T_{n-L}\left(z_{0}\right)\right)\right) \\
= & \left(p^{n} u_{1,0}+\zeta_{1} h_{n}(p, \mathbf{b}), q^{n} u_{2,0}+\zeta_{2} h_{n}(q, \mathbf{b})\right)
\end{aligned}
$$

and $s\left(T_{n}\left(z_{0}\right)\right)=b_{n}$, for all $n \geq 0$.

This is an important result because it is the mechanism we will use to prove that a given bitstream can be present at the output of a PDO. A similar result has been previously used in [20] to prove that under some circumstances the bitstream of a PDO, working with a resonator with only one resonance, is an $S^{3}$ sequence at the resonant frequency of the resonator.

Due to the presence of two different resonances in the resonator, one might expect a bitstream related to a mixture of both frequencies, most probably rendering the bitstream useless, from the point of view of obtaining at least one of the resonant frequencies directly from it. However, it will be proved that under some conditions, the bitstream at the output of the PDO with two resonances may be related only to one of them.

We will principally focus on a certain group of frequencies, called 'tuned' frequencies which were first found in [20]. The main reason to focus on these frequencies is that the oscillator with low or no losses presents a regularity pattern that simplifies the analysis of its dynamics.

\section{B. Lossless Resonator Case}

Now, we will obtain sufficient conditions for oscillation at one of the resonant frequencies of the resonator. This result will be valid for frequencies $f_{1}=\left(M_{1}\right) /\left(N_{1}\right)$ and $f_{2}=\left(M_{2}\right) /\left(N_{2}\right)$, with g.c.d. $\left(N_{1}, N_{2}\right)=1$, and g.c.d. $\left(M_{i}, N_{i}\right)=1$. Furthermore, we will assume that frequency $f_{1}$ is 'tuned' [20], i.e., for $\operatorname{sgn}\left(\zeta_{1}\right)=1$ :

$$
\begin{aligned}
& -N_{1} \text { even }:\left(\left\lfloor\frac{N_{1}}{4}+\frac{1}{2}\right\rfloor-(L+1) M_{1}\right) \bmod N_{1}=0 . \\
& -N_{1} \text { odd }:\left(\left\lfloor\frac{N_{1}}{2}+\frac{1}{2}\right\rfloor-(L+1) 2 M_{1}\right) \bmod 2 N_{1}=0 .
\end{aligned}
$$

In the case $\operatorname{sgn}\left(\zeta_{1}\right)=-1$, the former conditions are

$$
\begin{aligned}
& -N_{1} \text { even }:\left(\left\lfloor\frac{3 N_{1}}{4}+\frac{1}{2}\right\rfloor-(L+1) M_{1}\right) \bmod N_{1}=0 \\
& -N_{1} \text { odd }:\left(\left\lfloor\frac{3 N_{1}}{2}+\frac{1}{2}\right\rfloor-(L+1) 2 M_{1}\right) \bmod 2 N_{1}=0 .
\end{aligned}
$$

Under the above conditions it has been proved, [20], that if $p=e^{-j 2 \pi f}$, with $f$ being a tuned frequency and $b_{n}=$ $\operatorname{sgn}\left(\operatorname{Re}\left(p^{n} u_{0}\right)\right)$, for $u_{0} \in \mathbb{C}$, then

$$
\operatorname{sgn}\left(\operatorname{Re}\left(h_{n}(p, \mathbf{b})\right)\right)=b_{n}
$$

for $n \rightarrow \infty$, which is a result greatly simplifying the use of Lemma 1 for sequences of the form of (b). Now, we may state the following theorem:

Theorem 1: If an oscillator with two lossless resonances, $f_{1}=\left(M_{1}\right) /\left(N_{1}\right), p=e^{-j 2 \pi f_{1}}$, and $f_{2}=\left(M_{2}\right) /\left(N_{2}\right)$, $q=e^{-j 2 \pi f_{2}}$, with g.c.d. $\left(M_{i}, N_{i}\right)=1$, is tuned to $f_{1}$, and $u_{1,0}, u_{2,0} \in \mathbb{C}$ are such that $\left|\operatorname{Re}\left(p^{n} u_{1,0}\right)\right|>$ $\left|\beta u_{2,0}\right|+\left|\zeta_{1}\right| n \bmod N_{1}+\left|\beta \zeta_{2}\right| N_{1} N_{2}, 0 \leq n<N_{1}$, then $s\left(T_{n}\left(u_{1,0}, u_{2,0}, \operatorname{sgn}\left(\operatorname{Re}\left(p^{-1} u_{1,0}\right)\right), \ldots, \operatorname{sgn}\left(\operatorname{Re}\left(p^{-L} u_{1,0}\right)\right)\right)\right)=$ $\operatorname{sgn}\left(\operatorname{Re}\left(p^{n} u_{1,0}\right)\right), n \geq 0$. (The proof is given in Appendix I.)

This last result shows us that under some circumstances it is possible to obtain a bitstream at the output of the PDO related to only one of the resonances of the resonator. Among the conditions needed to ensure this oscillation we find the initial condition in both resonators. 

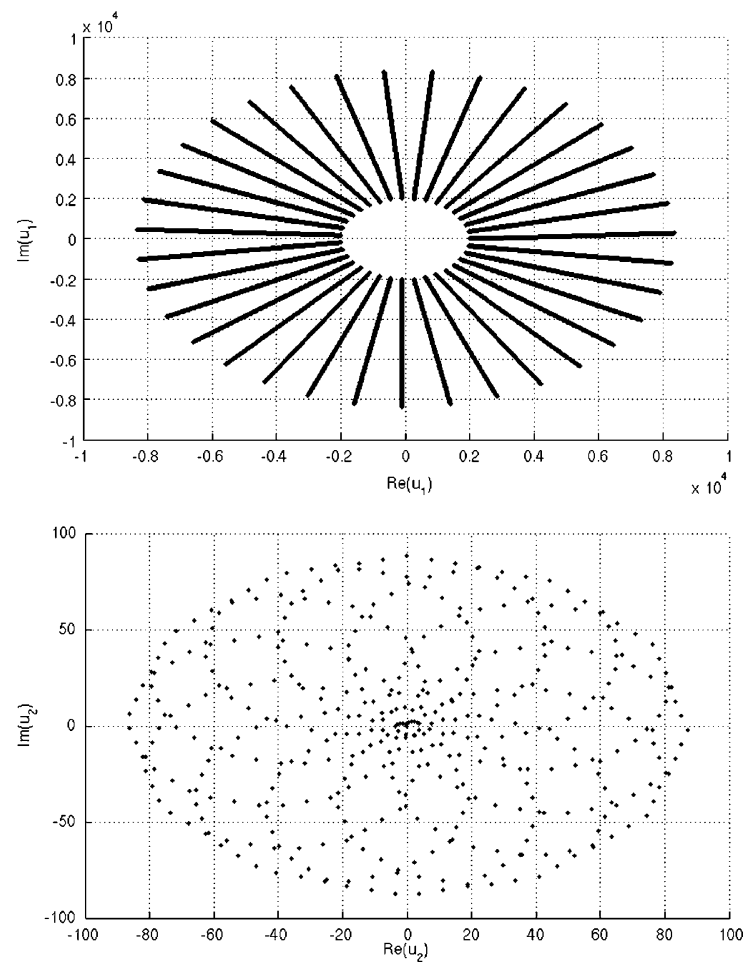

Fig. 5. Evolution of a PDO, with $L=2, \sigma=1$ and a lossless resonator with two tuned resonances, $f_{1}=3 / 35, f_{2}=1 / 12, \beta=1$. The initial conditions of both resonances are $u_{1,0}=1000 j e^{j \pi / 35}, u_{2,0}=10 j e^{j \pi / 6}$.

\section{Leaky Resonator Case}

A similar result may be found for the leaky case.

Theorem 2: For any oscillator with two leaky resonances such that $\left\langle f_{1}\right\rangle=\left(M_{1}\right) /\left(N_{1}\right)=(1) /(4(L+1)), p=e^{-j 2 \pi f_{1}}$ and $\left\langle f_{2}\right\rangle=\left(M_{2}\right) /\left(N_{2}\right), q=e^{-j 2 \pi f_{2}}, 0<\alpha_{p}, \alpha_{q}<1$, with g.c.d. $\left(M_{2}, N_{2}\right)=1$, g.c.d $\left(N_{1}, N_{2}\right)=1$, there exist a $n_{0}>0$ and a $\delta_{1}>0$ such that if $1-\delta_{1}<\alpha_{p}<1$ then there is a constant $C_{\alpha_{p}} \in \mathbb{R}^{+}$such that for $u_{1,0}, u_{2,0} \in \mathbb{C}$, with $\left|\operatorname{Re}\left(\alpha_{p}^{n} p^{n} u_{1,0}\right)\right|>C_{\alpha_{p}}+\left|\beta u_{2,0}\right|, 0 \leq n<n_{0}$, then $s\left(T_{n}\left(u_{1,0}, u_{2,0}, \operatorname{sgn}\left(\operatorname{Re}\left(p^{-1} u_{1,0}\right)\right), \ldots, \operatorname{sgn}\left(\operatorname{Re}\left(p^{-L} u_{1,0}\right)\right)\right)\right)=$ $\operatorname{sgn}\left(\operatorname{Re}\left(p^{n} u_{1,0}\right)\right), n \geq 0$. (The proof is given in Appendix II.)

This result extends the previous one on lossless resonances. It has been proved that under some conditions it is possible to obtain a bitstream related to only one of the resonances out of a PDO working with a leaky resonator.

\section{Discussion}

These results provide some insight into the fact that the oscillation frequency of a PDO working with more than one resonance generally may depend on the initial condition of the resonator. Let us assume that we have a resonator with two tuned frequencies. The above results state that, depending on the initial conditions of the resonator, the oscillation frequency will be one or the other. This is reflected in Figs. 5 and 6. As it can be observed, depending on the initial condition of the resonator, the oscillator may oscillate at the first or second resonance. This means that in order to control the selective activation of a given resonance we need a new mechanism: the 'anti-oscillation' mode.

The objective of the next section is to show that, depending on the normalized frequency of the resonator and the feedback filter of the PDO, either the PDO on average tends to provide energy
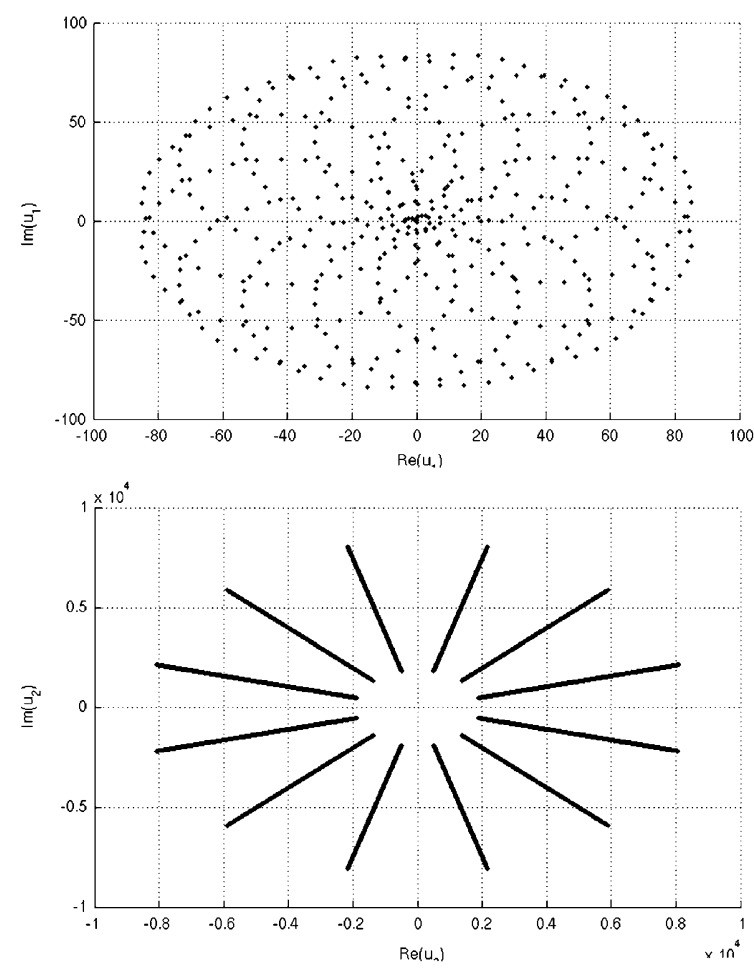

Fig. 6. Evolution of a PDO, with $L=2, \sigma=1$ and a lossless resonator with two tuned resonances, $f_{1}=3 / 35, f_{2}=1 / 12, \beta=1$. The initial conditions of both resonances are $u_{1,0}=10 j \mathrm{e}^{j \pi / 35}, u_{2,0}=1000 j \mathrm{e}^{j \pi / 6}$.

to the resonator, or, on the contrary, tends to extract it until a limit cycle is reached near the origin. This second behavior is called the 'anti-oscillation' mode. By choosing an appropriate feedback filter and a sampling frequency, it will be possible to put a given resonance in the "oscillation" mode and the others in the "anti-oscillation" mode. This mechanism will allow the selective activation of the vibration modes of the structure.

\section{Anti-Oscillation Mode}

\section{A. Simulation Results}

From the behavior observed experimentally and in simulations, given a configuration of the oscillator and a resonator two possible behaviors of the PDO are possible: either: 1) the oscillator generates a self-sustained oscillation in the resonator, or 2) it tends to cancel out any oscillation in the resonator.

In the first case, the excitation being applied to the resonator tends on average to introduce energy to the resonator, whereas in the second case, the excitation extracts energy from the resonator until a limit cycle is reached near the origin. The iterative map that we will consider is that of a PDO working with only one resonance:

$$
u_{n+1}=\alpha p u_{n}-j \sigma \zeta b_{n-L}, \quad n \geq 0
$$

where $p=e^{-j 2 \pi f}, f=\left(f_{0}\right) /\left(f_{S}\right) \sqrt{1-\rho^{2}}$ is the normalized resonant frequency, $\zeta$ is the normalized force, $L+1$ is the number of delays in the feedback loop $\left(G(z)=z^{-L-1}\right)$, and:

$$
\alpha=e^{-2 \pi \frac{\rho}{\sqrt{1-\rho^{2}}} f} \text {. }
$$

This iterative map is the same as that in (28), with parameters (25), except that the sign of the feedback loop, $\sigma$, is put in the map in explicit form. 


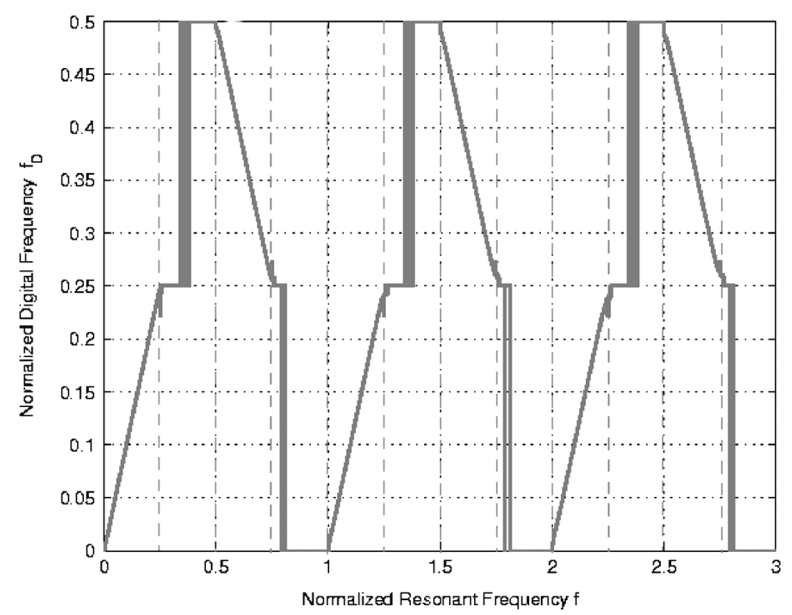

Fig. 7. Response of the oscillator, $L=1$, as a function of the normalized resonant frequency when no change of signs is made in the feedback loop ( $\sigma=$ 1 ), for a resonator with $\rho=0.0005$.

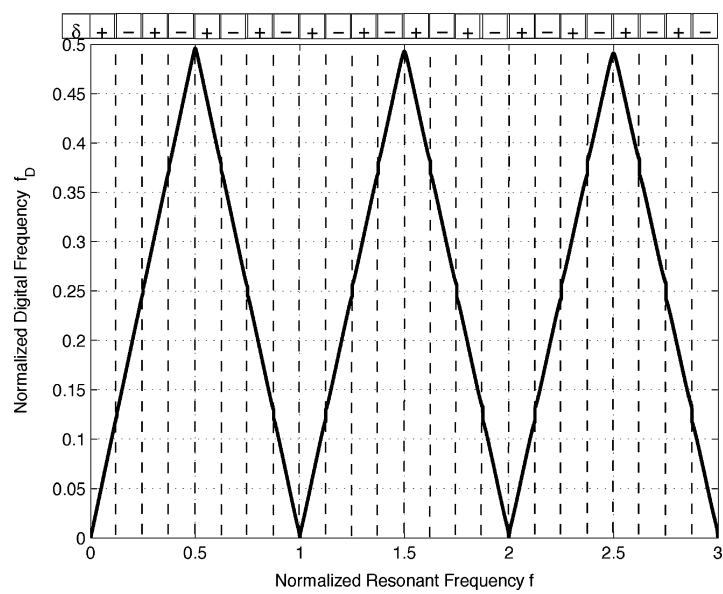

Fig. 8. Response of the oscillator, $L=3$, as a function of the normalized resonant frequency when the feedback sign is changed in the anti-oscillation regions, for a resonator with $\rho=0.0005$.

In all the simulations the oscillation frequency is obtained by generating an auxiliary bitstream. As pointed out in [20], any $S^{3}$ sequence can be converted to a first-order sigma-delta bitstream simply by edge detection. The new bitstream is generated by applying the following rule: $q_{n}=1 / 2\left|b_{n}-b_{n-1}\right|$. By filtering the oscillation frequency is now easily obtained. Although this result has been only proved for $S^{3}$ sequences, simulation as well as experimental results, [21], show that this procedure can be used in practical applications.

If the sign of the feedback filter is kept constant for all frequencies there are whole frequency segments where the oscillation frequency extracted from the bitstream is simply "wrong," in the sense that it is not related in any way to the resonant frequency of the resonator. This behavior can be observed in Fig. 7. As can be seen, there are some "forbidden" segments $(\langle f(L+1)\rangle \in[1 / 2,1))$ where the PDO does not follow the resonant frequency of the resonator [25], [37].

In order to obtain self-sustained oscillations for all frequencies it is necessary to change the sign of the feedback filter $(\sigma)$ in the forbidden frequency segments. Fig. 8 shows the oscillation frequency of a PDO, with $L=3$ and $\rho=0.0005$, in which the sign of the feedback loop is changed in the forbidden regions. Fig. 9 shows a similar result for an oscillator with $L=1$ and a

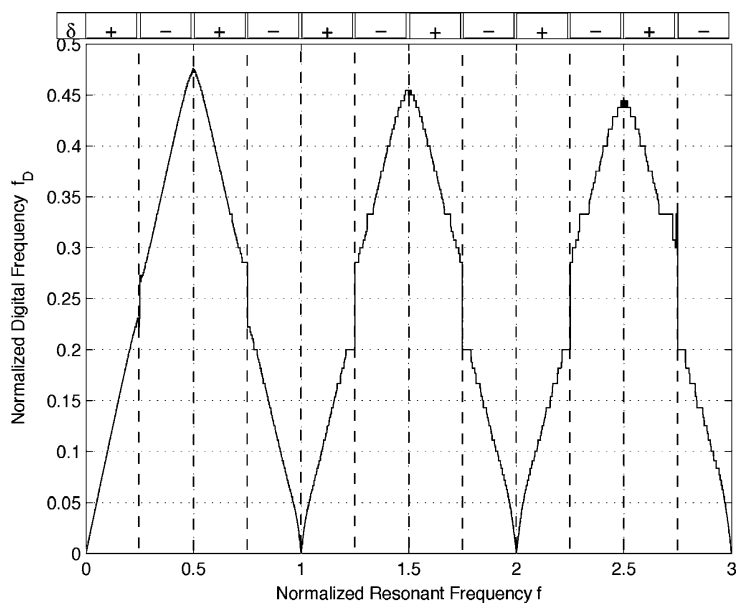

Fig. 9. Response of the oscillator, $L=1$, as a function of the normalized resonant frequency when the feedback sign is changed in the anti-oscillation regions, for a resonator with $\rho=0.01$.

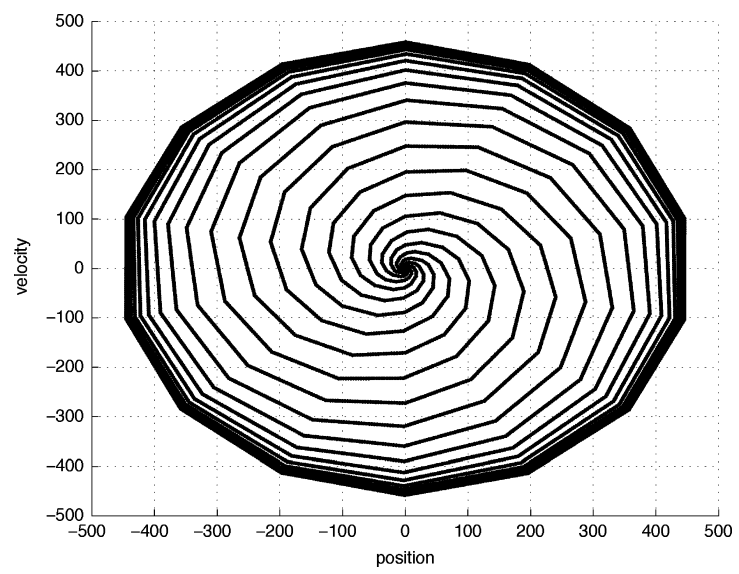

Fig. 10. Oscillation mode for a PDO with $f=1 / 7, \rho=0.0001, \sigma=-1$, $F=1, L=3$ and initial condition of the resonator $u_{0}=1+j$.

resonator with heavy losses $(\rho=0.01)$. As can be observed the oscillation frequency follows closely the resonant frequency of the resonator in the first case, whereas in the second case large differences can be observed due to the plateaus of the fractal response of the oscillator. The relation between the sign switching and the oscillation-anti-oscillation behavior will be shown in Section V-C.

In the anti-oscillation frequency segments the oscillator is on average extracting energy from the resonator. This effect can be seen in Figs. 10 and 11. In Fig. 10 the initial condition of the resonator is near the origin $\left(u_{0}=1+j\right)$. It is seen that the final orbit reaches an amplitude of approximately 450 . In this case the oscillator is working in the oscillation mode and it is on average always increasing the energy of the resonator. On the other hand, in Fig. 11, for the same frequency $f$ but changing the sign of the feedback loop and a large initial condition of the resonator $\left(u_{0}=50+50 j\right)$, the oscillator finally reaches an orbit near the origin. This means that the oscillator has extracted energy stored initially in the resonator until the limit cycle near the origin is reached.

\section{B. Regions With No Tuned Frequencies}

It was proved in [20] that a necessary condition to obtain self-sustained oscillations at the resonant frequency of a loss- 


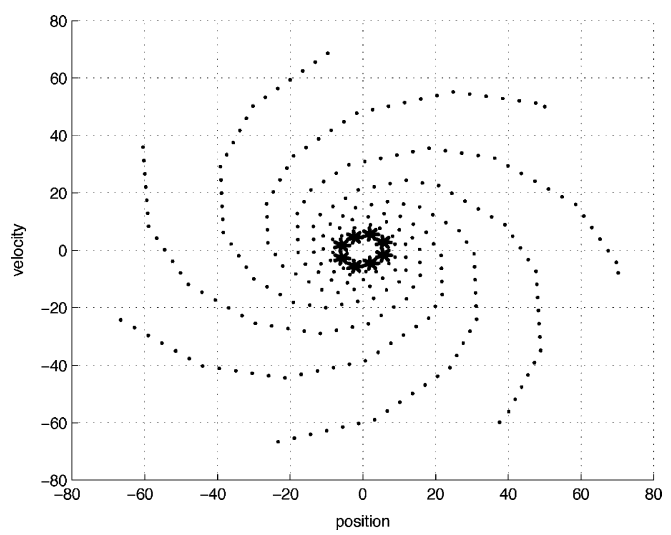

Fig. 11. Anti-oscillation mode for a PDO with $f=1 / 7, \rho=1 e-4, \sigma=1$, $F=1, L=3$ and initial condition of the resonator $u_{0}=50+50 j$.

less resonator is that the resonant frequency must be tuned. Extensive simulations have been carried out showing that the PDO does not generate oscillations in the frequency segments $\langle f(L+1)\rangle \in[0,1 / 2)$ when $\sigma>0$ and $\langle f(L+1)\rangle \in[1 / 2,1)$ when $\sigma<0$. The purpose of this section is to show that in these frequency segments there are no tuned frequencies and therefore it is not possible to find perfect $S^{3}$ sequences at the resonant frequency of the resonator in the lossless case.

Now, let us assume that $\sigma=+1$, then if a frequency $f=$ $M / N$, g.c.d. $(M, N)=1$, with $N$ even, is tuned there is an integer $m$ such that:

$$
m=\frac{\left\lfloor\frac{N}{4}+\frac{1}{2}\right\rfloor-(L+1) M}{N}
$$

which means that

$$
\left\langle(L+1) \frac{M}{N}\right\rangle=\frac{1}{4}+\frac{1}{2 N}-\frac{\langle N / 4+1 / 2\rangle}{N}
$$

and therefore, $\langle(L+1)(M / N)\rangle \notin((1 / 2), 1)$. Now, for $N$ odd, $m$ is

$$
m=\frac{\left\lfloor\frac{N}{2}+\frac{1}{2}\right\rfloor-(L+1) 2 M}{2 N}
$$

and therefore

$$
\left\langle(L+1) \frac{M}{N}\right\rangle=\frac{1}{4}+\frac{1}{4 N}
$$

which means that $\langle(L+1)(M / N)\rangle \in((1 / 4),(1 / 2)]$. In both cases, $N$ odd or even, we have that for any integer $k$ :

$$
\frac{M}{N} \notin\left(\frac{1}{2(L+1)}+\frac{k}{L+1}, \frac{1}{L+1}+\frac{k}{L+1}\right) .
$$

Following a similar procedure for the case $\sigma=-1$, it can be shown that $\langle(L+1)(M / N)\rangle \in[(1 / 2), 1]$ and therefore,

$$
\frac{M}{N} \notin\left(\frac{k}{L+1}, \frac{1}{2(L+1)}+\frac{k}{L+1}\right) .
$$

Taking into account [20], this result tells us that oscillations giving rise to perfect $S^{3}$ sequences at the resonant frequency of a lossless resonator are not possible in these regions. This is not a complete result because it does not provide any information regarding what happens in those forbidden zones, although it is completely consistent with the anti-oscillation modes that are always observed in simulations.

The above result, together with Proposition 2 of [20], shows that there are frequency segments on which an $S^{3}$ sequence at the resonant frequency of a lossless resonator is not possible.

\section{Energy Considerations for PDOs in the Antioscillation Mode}

It has been shown in [38] that under some conditions $S^{3}$ sequences at the resonant frequency of the resonator provide asymptotically a maximum in energy transfer to the resonator. The objective of this section is to show that, under some conditions, PDOs working in the anti-oscillation mode may provide maximum energy extraction from the resonator, i.e., absorption of the mechanical energy of the resonator into the electrical domain.

Each time a delta of force of amplitude $P$ is applied to a resonator at time $\tau=\tau_{n}$, the energy delivered to the resonator is (see the Appendix of [19]):

$$
\Delta E\left(\tau_{n}\right)=\frac{P^{2}}{2 m}+P x^{\prime}\left(\tau_{n}\right)
$$

where $m$ is the mass of the resonator and $x^{\prime}\left(\tau_{n}\right)$ is the resonator velocity at time $\tau=\tau_{n}$. This means that when a delta of force is applied to a resonator, depending on the sign and magnitude of both the excitation and the instantaneous velocity of the resonator, we may either increase or decrease its stored energy.

If we look again at the iterative map of a PDO with a lossless resonator, (39), if at a given time $n$ we have that:

$$
\operatorname{sgn}\left(\operatorname{Im}\left(p u_{n}\right)\right)=-\operatorname{sgn}(\sigma) b_{n-L}
$$

taking into account that $\operatorname{Im}\left(p u_{n}\right)=v_{n+1}-/ \omega_{0}$, i.e., the imaginary part of $p u_{n}$ is proportional to the instantaneous velocity of the resonator just before the delta is applied, then the energy transfer to the resonator at that sampling time has been maximum. In the case where $\operatorname{sgn}\left(\operatorname{Im}\left(p u_{n}\right)\right)=\operatorname{sgn}(\sigma) b_{n-L}$ the contrary happens, the energy extracted from the resonator has been maximum.

Let us first define the set of "anti-tuned" frequencies.

Definition 5: A rational frequency of the resonator, $f=$ $(M / N)$ is said to be an anti-tuned frequency of a PDO if either it obeys (36) and $\sigma<0$, or it obeys (37) and $\sigma>0$.

From the results of the previous section we know that no permanent oscillation at the resonant frequency is possible at any anti-tuned frequency. However it is possible to obtain a bitstream of the form $b_{n}=\operatorname{sgn}\left(\operatorname{Re}\left(p^{n} u_{0}\right)\right)$, out of any PDO, at least for some clock cycles, depending on the initial condition of the resonator.

Lemma 2: Given a PDO with a resonator and an initial condition, $u_{0}$, such that $\left|\operatorname{Re}\left(p^{n} u_{0}\right)\right|>n \zeta,\left|\operatorname{Im}\left(p^{n} u_{0}\right)\right|>n \zeta$, for $0 \leq n<n_{0}$, then $\operatorname{sgn}\left(\operatorname{Re}\left(u_{n}\right)\right)=\operatorname{sgn}\left(\operatorname{Re}\left(p^{n} u_{0}\right)\right)$ and $\operatorname{sgn}\left(\operatorname{Im}\left(p u_{n}\right)\right)=\operatorname{sgn}\left(\operatorname{Re}\left(-j p^{n+1} u_{0}\right)\right)$, for $0 \leq n<n_{0}$.

Proof: From the map of the oscillator, (39), we have that:

$$
u_{n}=p^{n} u_{0}-j \sigma \zeta p^{n-1} \sum_{i=0}^{n-1} p^{-i} b_{i-L}
$$

Now, if $\left|\operatorname{Re}\left(p^{n} u_{0}\right)\right|>n \zeta$ for $0 \leq n<n_{0}$, then $\operatorname{sgn}\left(\operatorname{Re}\left(u_{n}\right)\right)=\operatorname{sgn}\left(\operatorname{Re}\left(p^{\bar{n}} u_{0}\right)\right), \quad$ because $n \zeta>\left|\zeta p^{n-1} \sum_{i=0}^{n-1} p^{-i} b_{i-L}\right|$. On the other hand, 
from (49), it is easy to see that if $\left|\operatorname{Im}\left(p^{n} u_{0}\right)\right|>n \zeta$, for $0 \leq n<n_{0}$, then $\operatorname{sgn}\left(\operatorname{Im}\left(p u_{n}\right)\right)=\operatorname{sgn}\left(\operatorname{Re}\left(-j p^{n+1} u_{0}\right)\right)$.

This last result, means that for an unbounded set of initial conditions of the resonator, regardless of the configuration of the oscillator, the bitstream is dominated at least for some time directly by the initial condition of the resonator. Under these circumstances it is possible to study the energy efficiency of the PDO actuation while actuating the resonator, i.e., the number of times the sign of the delta of force applied to the resonator coincided with the sign of its velocity.

Definition 6: The energy efficiency, $\eta$, of a given finite-time pulsed actuation with amplitudes $b_{i}, 0 \leq i<n$ on a linear resonator with initial condition $u_{0}$ is defined as

$$
\eta=\frac{-\sigma}{n} \sum_{i=0}^{n-1} b_{i} \operatorname{sgn}\left(v_{i}\right)
$$

where $v_{i}$ is the velocity of the resonator at time $\tau=i T_{S}$ when the resonator is actuated with $n$ deltas with amplitudes $b_{i}, i=$ $0, \ldots, n-1$, and the initial condition of the resonator is $u_{0}$.

We will now analyze the energy efficiency of PDOs in antituned frequencies.

Theorem 3: Given a PDO with a lossless resonator with an anti-tuned frequency, $f=(M / N)$, if the initial condition, $u_{0}$, is such that $\left|\operatorname{Re}\left(p^{n} u_{0}\right)\right|>n \zeta,\left|\operatorname{Im}\left(p^{n} u_{0}\right)\right|>n \zeta$, for $0 \leq n<$ $n_{0} N+L$, then the energy efficiency of the actuation of the PDO from time $n=L$ to $n=L+n_{0} N$ on the resonator is

a) for $N$ even either $\eta=-1$ or $\eta=-(N-2) /(N)$;

b) for $N$ odd, either $\eta=-1$ or $\eta=-(N-1) / N)$.

Proof: The actuation the oscillator feeds into the resonator is $\operatorname{sgn}\left(\operatorname{Re}\left(u_{1, n-L}\right)\right)$. From Lemma 2, it follows that $\operatorname{sgn}\left(\operatorname{Re}\left(u_{n}\right)\right)=\operatorname{sgn}\left(\operatorname{Re}\left(p^{n} u_{0}\right)\right)$ and $q_{n}=\operatorname{sgn}\left(\operatorname{Im}\left(p u_{n}\right)\right)=$ $\operatorname{sgn}\left(\operatorname{Re}\left(-j p^{n+1} u_{0}\right)\right), 0 \leq n<n_{0} N$. If $L \leq n<n_{0} N+L$, then $b_{n-L}=\operatorname{sgn}\left(\operatorname{Re}\left(p^{n-L} u_{0}\right)\right)$.

We may distinguish three different cases:

a) $N=4 K, K \in \mathbb{N}$ : in this case $p^{-L-1}=-j$, and therefore $b_{n-L}=\operatorname{sgn}\left(\operatorname{Re}\left(p^{n+1-L-1} u_{0}\right)\right)=$ $\operatorname{sgn}\left(\operatorname{Re}\left(-j p^{n+1} u_{0}\right)\right)=\operatorname{sgn}\left(\operatorname{Im}\left(p^{n} u_{0}\right)\right)$. This means the the energy efficiency of this actuation is $\eta=-1$;

b) $N=4 K+2, K \in \mathbb{N}$ : then $p^{-L-1}=-j e^{j \pi / N}$. In this case, $b_{n-L}=\operatorname{sgn}\left(\operatorname{Re}\left(p^{n+1-L-1} u_{0}\right)\right)=$ $\operatorname{sgn}\left(\operatorname{Re}\left(-j e^{j \pi / N} p^{n+1} u_{0}\right)\right)$. Now, if $\left\langle\Theta\left(-j u_{0}\right) N\right\rangle \in$ $[0.5,1)$, where $\Theta(u)$ is defined as $\Theta(u)=1 / 4-$ $\arg (u) / 2 \pi$, then $\operatorname{sgn}\left(\operatorname{Re}\left(-j e^{j \pi / N} p^{n+1} u_{0}\right)\right)=$ $\operatorname{sgn}\left(\operatorname{Re}\left(-j p^{n+1} u_{0}\right)\right)$, and therefore $\eta=-1$. On the other hand, if $\left\langle\Theta\left(-j u_{0}\right) N\right\rangle \in[0,0.5)$, we will see that the difference between the sequences $\operatorname{sgn}\left(\operatorname{Re}\left(-j e^{j \pi / N} p^{n+1} u_{0}\right)\right)$ and $\operatorname{sgn}\left(\operatorname{Re}\left(-j p^{n+1} u_{0}\right)\right)$ is of two bits each $N$ bits, and therefore $\eta=-(N-2) /(N)$.

The $S^{3}$ sequence $q_{n}=\operatorname{sgn}\left(\operatorname{Re}\left(-j p^{n+1} u_{0}\right)\right)$ may be expressed in the following form:

$$
\begin{aligned}
& \operatorname{sgn}\left(\operatorname{Re}\left(-j p^{n+1} u_{0}\right)\right)=\left\langle(n+1) f-1 / 2+\Theta\left(-j u_{0}\right)\right\rangle \\
&-\left\langle(n+1) f+\Theta\left(-j u_{0}\right)\right\rangle \\
&=\left\langle\frac{(n+1) M-N / 2+\Theta\left(-j u_{0}\right) N}{N}\right\rangle \\
&-\left\langle\frac{(n+1) M+\Theta\left(-j u_{0}\right) N}{N}\right\rangle .
\end{aligned}
$$

Now, if the collection of sets $A_{n}$ is defined $A_{n}=\{0, \ldots, n-$ $1\}$, we may further define a function $I_{0}: \mathbb{Z} \rightarrow A_{N}$ as

$$
I_{0}(n)=\left\lfloor\left((n+1) M+\Theta\left(-j u_{0}\right)\right) N\right\rfloor \bmod N
$$

which allows us to rewrite the sequence $q_{n}$ as:

$$
\begin{aligned}
q_{n}= & \left\langle\frac{I_{0}(n)-N / 2+\left\langle\Theta\left(-j u_{0}\right) N\right\rangle}{N}\right\rangle \\
& -\left\langle\frac{I_{0}(n)+\left\langle\Theta\left(-j u_{0}\right) N\right\rangle}{N}\right\rangle \\
= & \left\langle\frac{I_{0}(n)-N / 2}{N}\right\rangle-\left\langle\frac{I_{0}(n)}{N}\right\rangle .
\end{aligned}
$$

Then, for any $n \in \mathbb{N}, q_{n}=1$ if $I_{0}(n \bmod N)<N / 2$, and $q_{n}=-1$ otherwise. On the other hand, the $S^{3}$ sequence $b_{n-L}=$ $\operatorname{sgn}\left(\operatorname{Re}\left(-j e^{j \pi / N} p^{n+1} u_{0}\right)\right)$ may be written as:

$$
\begin{array}{r}
b_{n-L}=\left\langle\frac{I_{0}(n)-N / 2-1 / 2+\left\langle\Theta\left(-j u_{0}\right) N\right\rangle}{N}\right\rangle \\
-\left\langle\frac{I_{0}(n)-1 / 2+\left\langle\Theta\left(-j u_{0}\right) N\right\rangle}{N}\right\rangle .
\end{array}
$$

It is clear that if $\left\langle\Theta\left(-j u_{0}\right) N\right\rangle \in[0.5,1)$ then both bitstreams are identical. If $\left\langle\Theta\left(-j u_{0}\right) N\right\rangle \in[0,0.5)$ then:

$$
\begin{aligned}
& b_{n-L}=\left.\frac{\left(I_{0}(n)-1\right)-N / 2+\left(1 / 2+\left\langle\Theta\left(-j u_{0}\right) N\right\rangle\right.}{N}\right\rangle \\
&-\left\langle\frac{\left(I_{0}(n)-1\right)+1 / 2+\left\langle\Theta\left(-j u_{0}\right) N\right\rangle}{N}\right\rangle \\
&=\left\langle\frac{\left(I_{0}(n)-1\right) \bmod N-N / 2}{N}\right\rangle \\
&-\left\langle\frac{\left(I_{0}(n)-1\right) \bmod N}{N}\right\rangle
\end{aligned}
$$

and therefore 2 in each $N$ bits change from the sequence $b_{n-L}$ with regard to $q_{n}$, which means that $\eta=(N-2) /(N)$.

$N$ odd: then $p^{-L-1}=-j e^{j \pi / 2 N}$, and therefore $b_{n-L}=$ $\operatorname{sgn}\left(\operatorname{Re}\left(-j e^{j \pi / 2 N} p^{n+1} u_{0}\right)\right)$. Now, if $\left\langle\Theta\left(-j u_{0}\right) 2 N\right\rangle \in[0.5,1)$ then $\operatorname{sgn}\left(\operatorname{Re}\left(-j e^{j \pi / 2 N} p^{n+1} u_{0}\right)\right)=\operatorname{sgn}\left(\operatorname{Re}\left(-j p^{n+1} u_{0}\right)\right)$ and therefore the energy efficiency is $\eta=-1$.

Now, the $S^{3}$ sequence $q_{n}=\operatorname{sgn}\left(\operatorname{Re}\left(-j p^{n+1} u_{0}\right)\right)$ may be expressed in the following form:

$$
\begin{aligned}
q_{n}= & \operatorname{sgn}\left(\operatorname{Re}\left(-j p^{n+1} u_{0}\right)\right) \\
= & \left\langle(n+1) f-1 / 2+\Theta\left(-j u_{0}\right)\right\rangle-\left\langle(n+1) f+\Theta\left(-j u_{0}\right)\right\rangle \\
= & \left\langle\frac{(n+1) 2 M-N+\Theta\left(-j u_{0}\right) 2 N}{2 N}\right\rangle \\
& -\left\langle\frac{(n+1) 2 M+\Theta\left(-j u_{0}\right) 2 N}{2 N}\right\rangle
\end{aligned}
$$

Following a similar procedure as before, we may define $I_{1}$ : $\mathbb{Z} \rightarrow A_{2 N}$ as

$$
I_{1}(n)=\left\lfloor\left((n+1) 2 M+\Theta\left(-j u_{0}\right)\right) 2 N\right\rfloor \bmod 2 N
$$


and therefore

$$
\begin{aligned}
q_{n}= & \left\langle\frac{I_{1}(n)-N+\left\langle\Theta\left(-j u_{0}\right) 2 N\right\rangle}{2 N}\right\rangle \\
& -\left\langle\frac{I_{1}(n)+\left\langle\Theta\left(-j u_{0}\right) 2 N\right\rangle}{2 N}\right\rangle \\
= & \left\langle\frac{I_{1}(n)-N}{2 N}\right\rangle-\left\langle\frac{I_{1}(n)}{2 N}\right\rangle
\end{aligned}
$$

which implies that if $I_{1}(n)<N$ then $q_{n}=1, q_{n}=-1$ otherwise. Now, if we look at the sequence $b_{n-L}$ :

$$
\begin{aligned}
b_{n-L}=\left\langle\frac{I_{1}(n)-}{N-1 / 2+\left\langle\Theta\left(-j u_{0}\right) 2 N\right\rangle}\right. & \\
- & \left\langle\frac{I_{1}(n)-1 / 2+\left\langle\Theta\left(-j u_{0}\right) 2 N\right\rangle}{2 N}\right\rangle
\end{aligned} .
$$

Once again it is clear that if $\left\langle\Theta\left(-j u_{0}\right) 2 N\right\rangle \in[0.5,1)$ then $q_{n}=b_{n-L}$, implying that $\eta=-1$. When $\left\langle\Theta\left(-j u_{0}\right) 2 N\right\rangle \notin$ $[0.5,1)$ :

$$
\begin{aligned}
b_{n-L}= & \left\langle\frac{\left(I_{1}(n)-1\right)-N+\left(1 / 2+\left\langle\Theta\left(-j u_{0}\right) 2 N\right\rangle\right.}{2 N}\right\rangle \\
& -\left\langle\frac{\left(I_{1}(n)-1\right)+1 / 2+\left\langle\Theta\left(-j u_{0}\right) 2 N\right\rangle}{2 N}\right\rangle \\
= & \left\langle\frac{\left(I_{1}(n)-1\right) \bmod 2 N-N}{2 N}\right\rangle \\
& -\left\langle\frac{\left(I_{1}(n)-1\right) \bmod 2 N}{2 N}\right\rangle
\end{aligned}
$$

and in this case it means that only one bit changes in $N$ bits between $q_{n}$ and $b_{n-L}$, because for any two $n_{1}, n_{2} \in \mathbb{Z}$ with $n_{1}-n_{2} \bmod N \neq 0$ it is $\left|I_{1}\left(n_{1}\right)-I_{1}\left(n_{2}\right)\right| \geq 2$. Now the energy efficiency is $\eta=-(N-1) /(N)$.

Corollary 1: Given a PDO with a lossless resonator with a tuned frequency, $f=(M / N)$, if the initial condition, $u_{0}$, is such that $\left|\operatorname{Re}\left(p^{n} u_{0}\right)\right|>n \zeta,\left|\operatorname{Im}\left(p^{n} u_{0}\right)\right|>n \zeta$, for $0 \leq n<$ $n_{0} N+L$, then the energy efficiency of the actuation of the PDO from time $n=L$ to $n=L+n_{0} N$ on the resonator is

a) for $N$ even either $\eta=1$ or $\eta=(N-2) /(N)$, and

b) for $N$ odd, either $\eta=1$ or $\eta=(N-1) /(N)$.

Proof: It follows step by step the proof of the previous result but taking into account that now the frequency is tuned, which means that either $\eta=1, \eta=(N-2) / N$ or $\eta=(N-$ 1) $/ N$, depending on whether $N$ is even or not, and the initial condition of the resonator.

Now, the following result can be proved for leaky resonators:

Theorem 4: For any $n_{0} \in \mathbb{N}$ and any anti-tuned frequency, $f=(M / N)$, there are some maximum losses, $0<\alpha_{0}<1$, such that for any PDO with a leaky resonator with $\alpha_{0}<|p|<1$, if the initial condition, $u_{0}$ obeys $\left|\operatorname{Re}\left(p^{n} u_{0}\right)\right|>\zeta\left(1-|p|^{n}\right) /(1-$ $|p|),\left|\operatorname{Im}\left(p^{n} u_{0}\right)\right|>\zeta\left(1-|p|^{n}\right) /(1-|p|)$, for $0 \leq n<n_{0} N+L$, then the energy efficiency of the actuation of the PDO from time $n=L$ to $n=L+n_{0} N$ on the resonator is:

a) for $N$ even either $\eta=-1$ or $\eta=-(N-2) /(N)$, and

b) for $N$ odd, either $\eta=-1$ or $\eta=-(N-1) /(N)$.
Proof: Due to the fact that $n_{0}$ is finite, it is possible to find $0<\alpha_{0}<1$, such that if $\alpha_{0}<|p|<1$ and $\left|\operatorname{Im}\left(p^{n} u_{0}\right)\right|>$ $\zeta\left(1-|p|^{n}\right) /(1-|p|)>0$ it is for $0 \leq n<n_{0} N+L$ :

$$
\operatorname{sgn}\left(x^{\prime}\left(n T_{S}{ }^{-}\right)\right)=\operatorname{sgn}\left(\operatorname{Im}\left(p^{n} u_{0}\right)-\rho \operatorname{Re}\left(p^{n} u_{0}\right)\right)
$$

or, equivalently

$\operatorname{sgn}\left(\operatorname{Im}\left(p^{n} u_{0}\right)-\frac{\rho}{\sqrt{1-\rho^{2}}} \operatorname{Re}\left(p^{n} u_{0}\right)\right)=\operatorname{sgn}\left(\operatorname{Im}\left(p^{n} u_{0}\right)\right)$.

Now, following a similar approach as in Lemma 2, if $\left|\operatorname{Re}\left(p^{n} u_{0}\right)\right|>\zeta\left(1-|p|^{n}\right) /(1-|p|),\left|\operatorname{Im}\left(p^{n} u_{0}\right)\right|>$ $\zeta\left(1-|p|^{n}\right) /(1-|p|)$, for $0 \leq n<n_{0} N+L$, then $\operatorname{sgn}\left(\operatorname{Re}\left(u_{n}\right)\right)=\operatorname{sgn}\left(p^{n} u_{0}\right)$ and $\operatorname{sgn}\left(\operatorname{Im}\left(p^{n} u_{0}\right)\right)=$ $\operatorname{sgn}\left(\operatorname{Re}\left(-j p^{n+1} u_{0}\right)\right), 0 \leq n<n_{0} N$. Finally following a similar procedure as before the result is obtained.

Corollary 2: For any $n_{0} \in \mathbb{N}$ and any tuned frequency, $f=$ $(M / N)$, there are some maximum losses, $0<\alpha_{0}<1$, such that for any PDO with a leaky resonator with $\alpha_{0}<|p|<1$, if the initial condition, $u_{0}$ obeys $\left|\operatorname{Re}\left(p^{n} u_{0}\right)\right|>\zeta\left(1-|p|^{n}\right) /(1-|p|)$, $\left|\operatorname{Im}\left(p^{n} u_{0}\right)\right|>\zeta\left(1-|p|^{n}\right) /(1-|p|)$, for $0 \leq n<n_{0} N+L$, then the energy efficiency of the actuation of the PDO from time $n=L$ to $n=L+n_{0} N$ on the resonator is:

a) for $N$ even either $\eta=1$ or $\eta=(N-2) /(N)$, and

b) for $N$ odd, either $\eta=1$ or $\eta=(N-1) /(N)$.

Proof: It follows step by step the proof of the previous result but taking into account that now the frequency is tuned.

\section{Characterization of the Anti-Oscillation Frequency Segments}

We have seen that if what governs the immediate evolution of the PDO is the initial condition of the oscillator, i.e., under the conditions of Lemma 2, the actuation of PDOs can produce extreme values of the energy efficiency, depending on whether the resonant frequency of the resonator is tuned or anti-tuned. The objective of this section is to establish a condition fully characterizing the anti-oscillation frequency segments.

Let us assume an initial condition of the resonator that guarantees a "natural evolution" of the resonator for $n_{0}+L$ clock cycles. In the case of a lossless resonator the condition might be $\left|\operatorname{Re}\left(p^{n} u_{0}\right)\right|>n \zeta,\left|\operatorname{Im}\left(p^{n} u_{0}\right)\right|>n \zeta$, for $0 \leq n<n_{0}+L$. In the case of a leaky resonator, $\left|\operatorname{Re}\left(p^{n} u_{0}\right)\right|>\zeta\left(1-|p|^{n}\right) /(1-$ $|p|),\left|\operatorname{Im}\left(p^{n} u_{0}\right)\right|>\zeta\left(1-|p|^{n}\right) /(1-|p|)$, for $0 \leq n<n_{0}+L$. Therefore, assuming the initial condition of the resonator is in these regions, we have that $b_{n-L}=\operatorname{sgn}\left(\operatorname{Re}\left(p^{n-L} u_{0}\right)\right)$ and $q_{n}=\operatorname{sgn}\left(\operatorname{Im}\left(p u_{n}\right)\right)=\operatorname{sgn}\left(\operatorname{Re}\left(-j p^{n+1} u_{0}\right), L \leq n<n_{0}+L\right.$. Therefore:

$$
\begin{aligned}
b_{n-L}= & \left\langle(n-L) f-\frac{1}{2}+\Theta\left(u_{0}\right)\right\rangle-\left\langle(n-L) f+\Theta\left(u_{0}\right)\right\rangle \\
= & \left\langle(n+1) f-(L+1) f-\frac{1}{2}+\Theta\left(u_{0}\right)\right\rangle \\
& -\left\langle(n+1) f-(L+1) f+\Theta\left(u_{0}\right)\right\rangle \\
q_{n}= & \left\langle(n+1) f-\frac{1}{2}+\frac{1}{4}+\Theta\left(u_{0}\right)\right\rangle \\
& -\left\langle(n+1) f+\frac{1}{4}+\Theta\left(u_{0}\right)\right\rangle
\end{aligned}
$$


Now, considering that $\sigma=1$, for $n_{0}$ large, in order to have $\eta \leq 0$, on average for all initial conditions, it must be $\langle(1 / 4)+$ $(L+1) f\rangle \in[0,1 / 4) \cup[3 / 4,1)$, which means that:

$$
\langle(L+1) f\rangle \in[0.5,1)
$$

and these are precisely the frequency segments where the antioscillation behavior is found (for $\sigma>0$ ). In case $\sigma<0$ then $\langle(L+1) f\rangle \in[0,0.5)$.

\section{CONCLUSION}

The dynamics of PDOs working with MEMS resonators with more than one resonance were analyzed. We started with a lumped model of coupled ordinary differential equations obtained for clamped-free beams. Each ODE is related to one of the resonances of the beam, which result from the decomposition in eigenmodes of the solution to the partial differential equation governing the motion of the beam. From this lumped model a set of iterative maps has obtained that serve to describe the dynamics of a PDO working with resonator with more than one resonance. It has been proved that under some circumstances PDOs can activate one of the resonances of the MEMS structure, but that this fact can depend on the initial condition of the resonator. In order to achieve an active control on the activation of the resonances another mechanism is necessary: the anti-oscillation mode. This mode has been analyzed and it has been shown that the energy efficiency of the actuation in the anti-oscillation frequency segments is negative. This mechanism will be used later in part II to actuate different resonant modes on a MEMS cantilever.

\section{APPENDIX I}

\section{PROOF OF THEOREM 1}

In order to obtain the proof of Theorem 1 we need first the following result:

Lemma 3: Given $M_{1}, M_{2}, N_{1}, N_{2} \in \mathbb{N}$, with g.c.d. $\left(N_{1}, N_{2}\right)=1$, and g.c.d. $\left(M_{i}, N_{i}\right)=1, i=0,1$, then

$$
\begin{aligned}
& \left\{\left(\left\langle k \frac{M_{1}}{N_{1}}\right\rangle,\left\langle k \frac{M_{2}}{N_{2}}\right\rangle\right): k=0, \ldots, N_{1} N_{2}-1\right\} \\
& \quad=\left\{\left(\frac{k}{N_{1}}, \frac{l}{N_{2}}\right): k=0, \ldots, N_{1}-1, l=0, \ldots, N_{2}-1\right\} .
\end{aligned}
$$

Proof: The result is evident for $N_{1}=N_{2}=1$. Now, for $N_{1} N_{2}>1$, let us assume that the first set does not have $N_{1} N_{2}$ elements, and therefore the above equality does not hold. This means that for a given $0<k_{0}<N_{1} N_{2}$ it is $\left\langle k_{0}\left(M_{1}\right) /\left(N_{1}\right)\right\rangle=\left\langle k_{0}\left(M_{2}\right) /\left(N_{2}\right)\right\rangle=0$, or what is the same, $k_{0} M_{1} \bmod N_{1}=k_{0} M_{2} \bmod N_{2}=0$. Now, taking into account that g.c.d. $\left(M_{i}, N_{i}\right)=1, i=0,1$, we have that $k_{0}=m_{1} N_{1}$ and $k_{0}=m_{2} N_{2}$, for some $m_{1}, m_{2} \in \mathbb{Z}$. On the other hand, because g.c.d. $\left(N_{1}, N_{2}\right)=1$, then $k_{0}=m_{0} N_{1} N_{2}$, with $m_{0} \in \mathbb{Z}$, and therefore $k_{0} \notin\left\{1, \ldots, N_{1} N_{2}-1\right\}$, which is a contradiction.

This only reflects the fact that $\mathbb{Z} / N_{1} \mathbb{Z} \times \mathbb{Z} / N_{2} \mathbb{Z}$ is a group isomorph to $\mathbb{Z} / N_{1} N_{2} \mathbb{Z}$ if and only if g.c.d. $\left(N_{1}, N_{2}\right)=1$.

This result allows us to bound the response of a given resonator, of resonant frequency $f_{2}=M_{2} / N_{2}$, to an $S^{3}$ sequence, of frequency $f_{1}=M_{1} / N_{1}$ of a different frequency, if their respective frequencies under the conditions of Lemma 3 , at times multiple of $N_{1} N_{2}$.

Lemma 4: For any binary sequence $\mathbf{b}_{u_{1,0}}=$ $\left(\operatorname{sgn}\left(\operatorname{Re}\left(p^{n} u_{1,0}\right)\right)\right)$, with $p=\exp \left(-j 2 \pi\left(M_{1}\right) /\left(N_{1}\right)\right), u_{1,0} \in$ $\mathbb{C}, \quad$ if $q=\exp \left(-j 2 \pi\left(M_{2}\right) /\left(N_{2}\right)\right)$, with g.c.d. $\left(N_{1}, N_{2}\right)=1$, and g.c.d. $\left(M_{i}, N_{i}\right)=1, i=0,1$, then $\left|h_{n}\left(q, \mathbf{b}_{u_{1,0}}\right)\right| \leq n \bmod N_{1} N_{2}$.

Proof: From Lemma 3, we have that

$$
\begin{aligned}
& \sum_{i=0}^{N_{1} N_{2}-1} e^{j 2 \pi \frac{M_{2}}{N_{2}} i} \operatorname{sgn}\left(\operatorname{Re}\left(e^{-j 2 \pi \frac{M_{1}}{N_{1}} i} u_{1,0}\right)\right) \\
& =\sum_{i=0}^{N_{2}-1} e^{j \frac{2 \pi i}{N_{2}}} \sum_{k=0}^{N_{1}-1} \operatorname{sgn}\left(\operatorname{Re}\left(e^{-j \frac{2 \pi k}{N_{1}}} u_{1,0}\right)\right)=0
\end{aligned}
$$

and therefore $h_{N_{1} N_{2}}\left(q, \mathbf{b}_{u_{1,0}}\right)=0$. Now, due to the fact that

$$
\begin{aligned}
h_{n}\left(q, \mathbf{b}_{u_{1,0}}\right)=\left\lfloor\frac{n}{N_{1} N_{2}}\right\rfloor \begin{array}{l}
h_{N_{1} N_{2}}\left(q, \mathbf{b}_{u_{1,0}}\right) \\
\\
+
\end{array} \quad h_{n \bmod N_{1} N_{2}}\left(q, \mathbf{b}_{u_{1,0}}\right) .
\end{aligned}
$$

which means that $\left|h_{n}\left(q, \mathbf{b}_{u_{1,0}}\right)\right| \leq n \bmod N_{1} N_{2}$.

Now, we may proceed with proving Theorem 1 :

Proof of Theorem 1: Following a similar approach to that used for proving Theorem 2 in [20], it can be shown that for a binary sequence $\mathbf{b}_{u_{1,0}}=\operatorname{sgn}\left(p^{n} u_{1,0}\right)$ we have that

$$
h_{n}\left(p, \mathbf{b}_{u_{1,0}}\right)=h_{n \bmod N_{1}}\left(p, u_{1,0}\right)+\left\lfloor\frac{n}{N_{1}}\right\rfloor p^{n} h_{N_{1}}\left(p, u_{1,0}\right) .
$$

Now, due to the fact that $f_{1}$ is a tuned frequency, it is $\operatorname{sgn}\left(\operatorname{Re}\left(p^{n} h_{N_{1}}\left(p, u_{1,0}\right)\right)\right)=\operatorname{sgn}\left(\operatorname{Re}\left(p^{n} u_{1,0}\right)\right)$ or $\operatorname{sgn}\left(\operatorname{Re}\left(p^{n} h_{N_{1}}\left(p, u_{1,0}\right)\right)\right)=0, n \geq 0$. On other hand, retaking Lemma 5, we have that $\left|h_{n}\left(q, \mathbf{b}_{u_{1,0}}\right)\right| \leq n \bmod N_{1} N_{2}$. Therefore for a sufficiently large $n_{0}$, it is for $n>n_{0}$, $\operatorname{sgn}\left(\operatorname{Re}\left(p^{n} u_{1,0}\right)\right)=\operatorname{sgn}\left(\operatorname{Re}\left(\zeta_{1} h_{n}\left(p, \mathbf{b}_{u_{1,0}}\right)\right)\right.$, and

$$
\mid \operatorname{Re}\left(\zeta_{1} h_{n}\left(p, \mathbf{b}_{u_{1,0}}\right)|>| \beta \operatorname{Re}\left(q^{n} u_{2,0}+\zeta_{2} h_{n}\left(q, \mathbf{b}_{u_{1,0}}\right)\right) \mid\right.
$$

and therefore

$$
\begin{aligned}
& \operatorname{sgn}\left(\operatorname { R e } \left(p^{n} u_{1,0}+\zeta_{1} h_{n}\left(p, \mathbf{b}_{u_{1,0}}\right)\right.\right. \\
& \left.\left.+\beta\left(q^{n} u_{2,0}+\zeta_{2} h_{n}\left(q, \mathbf{b}_{u_{1,0}}\right)\right)\right)\right) \\
& \quad=\operatorname{sgn}\left(\operatorname{Re}\left(p^{n} u_{1,0}\right)\right), \quad n>n_{0}
\end{aligned}
$$

Finally, if $\left|\operatorname{Re}\left(p^{n} u_{1,0}\right)\right|>\left|\beta u_{2,0}\right|+\left|\zeta_{1}\right| n \bmod N_{1}+$ $\left|\beta \zeta_{2}\right| N_{1} N_{2}, 0 \leq n<N_{1}$, then (72) is also true for $n \geq 0$, and therefore Lemma 1 can be applied.

\section{APPENDIX II \\ PROOF OF THEOREM 2}

We begin with an auxiliary result.

Lemma 5: Given a binary sequence $\mathbf{b}_{u_{1,0}}=$ $\left(\operatorname{sgn}\left(\operatorname{Re}\left(p^{n} u_{1,0}\right)\right)\right)$, with $p=\exp \left(-j 2 \pi\left(M_{1}\right) /\left(N_{1}\right)\right), u_{1,0} \in$ 
$\mathbb{C}$ and $q=\exp \left(-j 2 \pi\left(M_{2}\right) /\left(N_{2}\right)\right)$, with g.c.d. $\left(N_{1}, N_{2}\right)=1$, and g.c.d. $\left(M_{i}, N_{i}\right)=1, i=0,1$, for any $\epsilon>0$ there is a $\delta>0$ such that for $1-\delta<\alpha<1$ :

$$
\left|h_{n}\left(\alpha q, \mathbf{b}_{u_{1,0}}\right)\right| \leq \frac{1-\alpha^{n \bmod N_{1} N_{2}}}{1-\alpha}+\epsilon
$$

Proof: For any $n \geq 0$

$$
\begin{aligned}
h_{n}\left(\alpha q, \mathbf{b}_{u_{1,0}}\right)= & h_{n \bmod N_{1} N_{2}}\left(\alpha q, \mathbf{b}_{u_{1,0}}\right)+\frac{1-\alpha^{\left\lfloor\frac{n}{N_{1} N_{2}}\right\rfloor N_{1} N_{2}}}{1-\alpha^{N_{1} N_{2}}} \\
& \times \alpha^{n \bmod N_{1} N_{2}} q^{n} h_{N_{1} N_{2}}\left(\alpha q, \mathbf{b}_{u_{1,0}}\right)
\end{aligned}
$$

Now, due to the continuity of $h_{N_{1} N_{2}}\left(\alpha q, \mathbf{b}_{u_{1,0}}\right)$ as a function of $\alpha$, and taking into account that $h_{N_{1} N_{2}}\left(q, \mathbf{b}_{u_{1,0}}\right)=0$, then for $\alpha \rightarrow 1$ we have that $h_{N_{1} N_{2}}\left(\alpha q, \mathbf{b}_{u_{1,0}}\right) \rightarrow 0$, and the result is easily obtained.

Corollary 3: If to the conditions of Lemma 5 we add that $\left\langle M_{1} / N_{1}\right\rangle \neq 0$ or $\left\langle M_{2} / N_{2}\right\rangle \neq 0$ then there is a $\delta>0$ such that for $1-\delta<\alpha<1$ :

$$
\left|h_{n}\left(\alpha q, \mathbf{b}_{u_{1,0}}\right)\right|<\frac{1-\alpha^{n \bmod N_{1} N_{2}}}{1-\alpha}
$$

With the above results we may proceed to prove the second theorem:

Proof of Theorem 2: For any $n \geq 0$ we have that:

$$
h_{n}\left(\alpha_{p} p, \mathbf{b}_{u_{1,0}}\right)=h_{n \bmod N_{1} N_{2}}\left(\alpha_{p} p, \mathbf{b}_{u_{1,0}}\right)+a_{n} p^{n} h_{N_{1} N_{2}}\left(\alpha_{p} p, \mathbf{b}_{u_{1,0}}\right)
$$

where $a_{n}$ is:

$$
a_{n}=\frac{1-\alpha_{p}^{\left\lfloor\frac{n}{N_{1} N_{2}}\right\rfloor N_{1} N_{2}}}{1-\alpha_{p}^{N_{1} N_{2}}} \alpha_{p}^{n \bmod N_{1} N_{2}}
$$

which means that:

$$
\lim _{n \rightarrow \infty, \alpha \rightarrow 1} \frac{1}{a_{n}} h_{n}\left(\alpha_{p} p, \mathbf{b}_{u_{1,0}}\right)=p^{n} h_{N_{1} N_{2}}\left(p, \mathbf{b}_{u_{1,0}}\right)
$$

Now, taking into account that for $\left\langle f_{1}\right\rangle=(1) /(4(L+1))$ :

$$
\frac{\left\langle\left(\frac{1}{4}-\arg \left(h_{N_{1} N_{2}}\left(p, \mathbf{b}_{u_{1,0}}\right)\right) / 2 \pi\right) N_{1}\right\rangle}{N_{1}}=\frac{1}{2 N_{1}} \neq 0
$$

then, because $h_{N_{1} N_{2}}\left(\alpha_{p} p, \mathbf{b}_{u_{1,0}}\right)$ is continuous as a function of $\alpha_{p}$, there is a $\delta_{0}>0$ such that for $\alpha_{p} \in\left(1-\delta_{0}, 1+\delta_{0}\right)$, we have that $\operatorname{sgn}\left(\operatorname{Re}\left(p^{n} h_{N_{1} N_{2}}\left(\alpha_{p} p, \mathbf{b}_{u_{1,0}}\right)\right)\right)=\operatorname{sgn}\left(\operatorname{Re}\left(p^{n} u_{1,0}\right)\right), n \geq$ 0 . Finally, taking into account expression (77) and Lemma 5 , there exists a $0<\delta_{1} \leq \delta_{0}$ such that for $1>\alpha_{p}>1-\delta_{1}$ and a sufficiently high $n_{0}$ it is for $n>n_{0}$ :

$$
\begin{aligned}
& \left|\operatorname{Re}\left(\frac{\left.1-\alpha_{p}^{L \frac{n}{N_{1} N_{2}}}\right\rfloor N_{1} N_{2}}{1-\alpha_{p}^{N_{1} N_{2}}} \alpha_{p}^{n \bmod N_{1} N_{2}} p^{n} h_{N_{1} N_{2}}\left(\alpha_{p} p, \mathbf{b}_{u_{1,0}}\right)\right)\right| \\
& \quad>\frac{1-\alpha_{p}^{n \bmod N_{1} N_{2}}}{1-\alpha_{p}}+|\beta| \frac{1-\alpha_{q}^{n \bmod N_{1} N_{2}}}{1-\alpha_{q}} \\
& \quad \geq\left|h_{n \bmod N_{1} N_{2}}\left(\alpha_{p} p, \mathbf{b}_{u_{1,0}}\right)\right|+\left|\beta h_{n}\left(\alpha_{q} q, \mathbf{b}_{u_{1,0}}\right)\right|
\end{aligned}
$$

which means that $\operatorname{sgn}\left(\operatorname{Re}\left(h_{n}\left(\alpha_{p} p, \mathbf{b}_{u_{1,0}}\right)+\beta \alpha_{q}^{n} q^{n} u_{2,0}+\right.\right.$ $\left.\left.\beta h_{n}\left(\alpha_{q} q, \mathbf{b}_{u_{1,0}}\right)\right)\right)=\operatorname{sgn}\left(\operatorname{Re}\left(p^{n} u_{1,0}\right)\right)$ for $n>n_{0}$.

Finally, if $\left|\operatorname{Re}\left(\alpha_{p} p^{n} u_{1,0}\right)\right|>C_{\alpha_{p}}+\left|\beta u_{2,0}\right|$, for $0 \leq n \leq n_{0}$, where:

$$
C_{\alpha_{p}}=\frac{1-\alpha_{p}^{N_{1} N_{2}}}{1-\alpha_{p}}+|\beta| \frac{1-\alpha_{q}^{N_{1} N_{2}}}{1-\alpha_{q}}
$$

then $\operatorname{sgn}\left(\operatorname{Re}\left(h_{n}\left(\alpha_{p} p, \mathbf{b}_{u_{1,0}}\right)+\beta \alpha_{q} q^{n} u_{2,0}+\right.\right.$ $\left.\left.\beta h_{n}\left(\alpha_{q} q, \mathbf{b}_{u_{1,0}}\right)\right)\right)=\operatorname{sgn}\left(\operatorname{Re}\left(p^{n} u_{1,0}\right)\right)$ for $n \geq 0$, and therefore Lemma 1 can be applied.

\section{REFERENCES}

[1] S. D. Senturia, Microsystem Design.. New York: Springer, 2004.

[2] K. B. Brown, W. Allegretto, F. E. Vermeulen, and A. M. Robinson, "Simple resonating microstructures for gas pressure measuremnets," $J$. Micromechan. Microeng., vol. 12, pp. 204-210, 2002.

[3] V. Cimalla, F. Niebelschütz, K. Tonisch, C. Foerster, K. Brueckner, I. Cimalla, T. Friedrich, J. Pezoldt, R. Stephan, M. Hein, and O. Ambacher, "Nanoelectromechanical devices for sensing applications," Sensors and Actuators B, vol. 126, pp. 24-34, 2007.

[4] G. Keskar, B. Elliott, J. Gaillard, M. J. Skove, and A. M. Rao, "Using electric actuation and detection of oscillations in microcantilevers for pressure measurements," Sensors and Actuators A, vol. 147, pp. 203-209, 2008.

[5] R. Legtenberg and H. A. C. Tilmans, "Electrostatically driven vacuumencapsulated polysilicon resonators. Part I. Design and fabrication," Sensors and Actuators A, vol. 45, pp. 57-66, 1994.

[6] A. Fargas Marquès, R. C. Castello, and A. M. Shkel, Modelling the Electrostatic Actuation of MEMS: State of the Art 2005 Univ. Politècnica de Catalunya, Spain, Tech. Rep. IOC-DT-P-2005-18, Sep. 2005.

[7] G. K. Fedder and R. T. Howe, "Multimode digital control of a suspended polysilicon microstructure," J. Microelectromech. Syst., vol. 5, no. 4, pp. 283-297, Dec. 1996.

[8] T. Ono and M. Esashi, "Mass sensing with resonating ultra-thin silicon beams detected by a double-beam laser Doppler vibrometer," Meas. Sci. Technol., vol. 15, pp. 1977-1981, 2004.

[9] W. Zhang, R. Baskaran, and K. L. Turner, "Effect of cubic nonlinearity on auto-parametrically amplified resonant MEMS mass sensor," Sensors and Actuators A, vol. 102, pp. 139-150, 2002.

[10] W. Zhang and K. L. Turner, "Noise analysis in parametric resonance based mass sensing," in Proc. 2004 ASME Int. Mechan, Eng, Congress and Expo., Anaheim, CA, Nov. 13-20, 2004.

[11] W. Zhang and K. L. Turner, "Application of parametric resonance amplification in a single-crystal silicon micro-oscillator based mass sensor," Sensors and Actuators A, vol. 122, pp. 23-30, 2005.

[12] L. A. Oropeza-Ramos, C. B. Burgner, and K. L. Turner, "Robust microrate sensor actuated by parametric resonancerobust micro-rate sensor actuated by parametric resonance," Sensors and Actuators A, vol. 152, pp. 80-87, 2009.

[13] C. Acar and A. M. Shkel, "Structural design and experimental characterization of torsional micromachined gyroscopes with non-resonant drive mode," J. Micromechan.Microeg., vol. 14, pp. 15-25, 2004.

[14] T. Wu, W. Chang, and J. Hsu, "Effect of tip length and normal and lateral contact stifness on the flexural vibration responses of atomic force microscope cantilevers," Microelectron. Eng., vol. 71, pp. 15-20, 2004.

[15] W. Chang, H. Lee, and T. Y. Chen, "Study of the sensitivity of the first four flexural modes of an AFM cantilever with a sidewall probe," Ultramicroscopy, vol. 108, pp. 619-624, 2008.

[16] J. Sanchez-Rojas et al., "Piezoelectric modal sensors/actuators based on microplates applying surface electrode patterning," in Proc. 15th Int. Conf. on Solid-State Sensors, Actuators and Microsystems (TRANSDUCERS-09), Denver, CO, USA, June 21-25, 2009.

[17] X. Xia and X. Lin, "Resonance-mode effect on microcantilever masssensing performance in air," Rev. Scientific Instrum., vol. 79, p. 074301, 2008.

[18] M. Domínguez, J. Pons-Nin, J. Ricart, A. Bermejo, and E. Figueras Costa, "A novel $\Sigma-\Delta$ pulsed digital oscillator (PDO) for MEMS," IEEE Sensors J., vol. 5, pp. 1379-1388, Dec. 2005. 
[19] M. Domínguez, J. Pons-Nin, J. Ricart, A. Bermejo, E. Figueras Costa, and M. Morata, "Analysis of the $\Sigma-\Delta$ pulsed digital oscillator for MEMS,” IEEE Trans. Circuits Syst. I, Regular Papers, vol. 52, no. 11, pp. 2286-2297, Nov. 2005.

[20] M. Domínguez, J. Pons-Nin, and J. Ricart, "General dynamics of pulsed digital oscillators," IEEE Trans. Circuits Syst. I, Reg. Papers, vol. 55, no. , pp. 2038-2050, 2008

[21] J. Ricart, J. Pons, M. Domínguez, A. Rodríguez, E. Figueras, M. C. H. J. Gutiérrez, and I. Sayago, "Application of pulsed digital oscillators to volatile organic compounds sensing," Sensors and Actuators B, vol. 134, pp. 773-779, 2008.

[22] J. A. Pelesko and D. H. Bernstein, Modeling of MEMS and NEMS. London, U.K.: Chapman\&Hall, 2003.

[23] A. H. Nayfeh, M. I. Younis, and E. M. Abdel-Rahman, "Reduced-order models for MEMS applications," Nonlinear Dyn., vol. 41, pp. 211-236, 2005.

[24] J. Wu and L. R. Carley, "Electromechanical $\Delta \Sigma$ modulation with high-Q micromechanical accelerometers and pulse density modulated force feedback," IEEE Trans. Circuits Syst. I, Reg. Papers, vol. 53, no. 3, pp. 274-287, Mar. 2006.

[25] M. Domínguez, J. Pons-Nin, and J. Ricart, "Application of pulsed digital oscillators in 'reverse mode' to eliminate undesired vibrations in high-Q MEMS resonators," in Proc. IEEE International Symposium on Circuits and Systems 2007, New Orleans, USA, May 27-30, 2007, pp. $925-928$.

[26] A. Teplinsky and O. Feely, "Limit cycles in a MEMS oscillator," IEEE Trans. Circuits Syst. II, Expr. Briefs, vol. 55, no. 9, pp. 882-886, Sep. 2008.

[27] E. Blokhina and O. Feely, "A kicked oscillator as a model of a pulsed MEMS system,” Int. J. Bifurc. Chaos, vol. 19, no. 1, pp. 187-202, 2009.

[28] K. Graff, Wave Motion in Elastic Solids. New York: Dover, 1975.

[29] H. A. C. Tilmans and R. Legtenberg, "Electrostatically driven vacuumencapsulated polysilicon resonators. Part II. Theory and performance," Sensors and Actuators A, vol. 45, pp. 67-84, 1994.

[30] E. M. Abdel-Rahman, M. I. Younis, and A. H. Nayfeh, "Characterization of the mechanical behavior of an electrically actuated microbeam," J. Micromechan. Microeng., vol. 12, pp. 759-766, 2002.

[31] M. I. Younis and A. H. Nayfeh, "A study of the nonlinear response of a resonant microbeam to an electric actuation," Nonlinear Dyn., vol. 31, pp. 91-117, 2003.

[32] M. I. Younis, E. M. Abdel-Rahman, and A. Nayfeh, "A reduced-order model for electrically actuated microbeam-based MEMS," J. Microelectromech. Syst., vol. 12, no. 5, pp. 672-680, Oct. 2003.

[33] W. C. Xie, H. P. Lee, and S. P. Lim, "Nonlinear dynamic analysis of MEMS switches by nonlinear modal analysis," Nonlinear Dynamics, vol. 31, pp. 243-256, 2003.

[34] Y. C. Liang, W. Z. Lin, H. P. Lee, S. P. Lim, K. H. Lee, and H. Sun, "Proper orthogonal decomposition and its applications-Part II: Model reduction for MEMS dynamical analysis," J. Sound Vibration, vol. 256, pp. 515-532, 2002.

[35] G. K. Fedder, "Simulation of microelectromechanical systems," Ph.D. dissertation, Univ. California, Berkeley, 1994.

[36] M. Domínguez, J. Pons-Nin, J. Ricart, and E. Figueras, "The MEMS pulsed digital oscillator (PDO) below the Nyquist limit," Sensors and Actuators A, vol. 136, pp. 690-696, 2007.

[37] E. Blokhina, O. Feely, and M. Dominguez, "Dynamics of the MEMS pulsed digital oscillator with multiple delays in the feedback loop," in Proc. of IEEE Int. Symp. on Circuits and Systems (ISCAS) 2009, pp. 1903-1906.

[38] M. Domínguez, "Energy efficiency of pulsed actuations on linear resonators," IEEE Trans. Circuits Syst. I, vol. 56, no. 12, pp. 2678-2688, Dec. 2009.

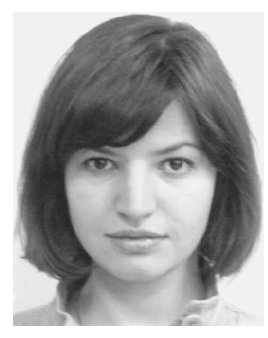

Elena Blokhina (S'05-M'06) received the M.S. and $\mathrm{Ph} . \mathrm{D}$. degrees in physics from Saratov State University, Russia, in 2002 and 2005, respectively.

From 2005 to 2007 she was a research scientist in Saratov State University where her project concerned nonlinear dynamics and chaos in microwave electron devices. She is currently a postdoctoral researcher in the Circuit and Systems Research Group at University College Dublin. Her research interests include nonlinear dynamics and oscillation theory and their application to the vibration analysis of MEMS.

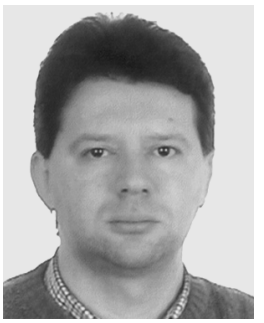

Joan Pons-Nin received the M.Sc. and Ph.D. degrees in electronic engineering from the Universitat Politècnica de Catalunya, Barcelona, Spain, in 1985 and 1995, respectively.

He is an Associate Professor with the Department of Electronic Engineering at the Telecommunications Engineering School of Barcelona, Spain. . $\mathrm{He}$ is currently working on oscillators for MEMS resonators. He worked also on emitter characterization and optimization for advanced bipolar devices and polysilicon contacted silicon solar cells. Other research topics of his interest are semiconductor devices simulation and digital control circuitry.

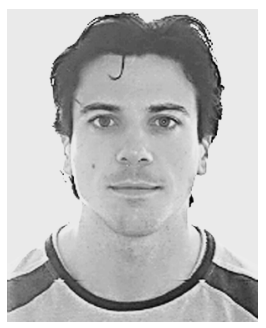

Jordi Ricart received the M.Sc. degree in Ingeniería Electrónica from the Universitat Politécnica de Catalunya (UPC), Barcelona, Spain, in 2004. He joined the Micro and Nano Technologies Research Group of the UPC both as a Research Assistant and as a Ph.D. degree student.

$\mathrm{He}$ is actively working in sensors for space applications and nonlinear circuits for MEMS applications, such as oscillators.

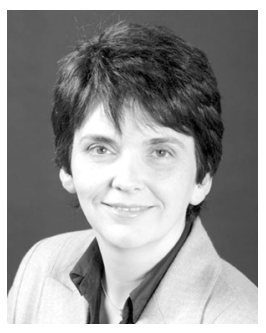

Orla Feely (S'85-M'86-SM'87-F'09) received the B.E. degree in electronic engineering from University College Dublin in 1986, and the M.S. and Ph.D. degrees in electrical engineering from the University of California, Berkeley, in 1990 and 1992, respectively.

She is currently a Professor in the School of Electrical, Electronic and Mechanical Engineering, University College Dublin. Her research interests lie in the area of nonlinear dynamics of electronic circuits.

Prof. Feely received the Best Paper Award of the International Journal of Circuit Theory and Applications in 2007, and the Best Paper Award of the European Conference on Circuit Theory and Design in 1997. Her Ph.D. thesis on sigma-delta modulation won the D. J. Sakrison Memorial Prize for outstanding and innovative research, awarded annually by the Department of Electrical Engineering and Computer Sciences at U. C. Berkeley. She has served as Associate Editor of the IEEE TRANSACTIONS ON Circuits AND Systems PART I: Fundamental THEORY AND APPLICATIONS, and is a member of the Editorial Board of the International Journal of Circuit Theory and Applications.

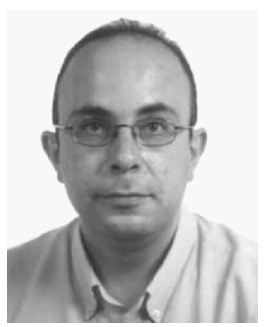

Manuel Dominguez Pumar (M'99) received the M.Sc. and Ph.D. degrees in electronic engineering from the Universitat Politècnica de Catalunya (UPC), Barcelona, Spain, in 1994 and 1997, respectively, and a Degree in mathematics from the Universidad Nacional de Educación a Distancia (UNED), Madrid, Spain, with honors., in 2005.

He has been with the UPC Department of Electronic Engineering since 1994, where he is now Associate Professor. He was visiting scholar at the Courant Institute of Mathematical Sciences from September 2006 to August 2007. His research areas include the design and development of oscillators, MEMS sensors and actuators, sensors for space applications, sigma-delta modulation applied to MEMS and non-linear circuits in general. 